\title{
Chronologie du site moustérien de type Quina des Pradelles (Marillac-le-Franc, Charente, France)
}

Marine Frouin, Christelle Lahaye, Norbert Mercier, Pierre Guibert, Isabelle Couchoud, Jean-Pierre Texier, Aurélien Royer, Sandrine Costamagno, Cédric Beauval, Laurence Bourguignon, Liliane Meignen, Jean-Guillaume Bordes, Guillaume Seguin, Hervé Bocherens, François LacrampeCuyaubère, Xavier Muth, Eva-Maria Geigl, Rainer Grün, María Dolores Garralda, Bernard Vandermeersch, Alan E. Mann et Bruno Maureille

\section{(2) OpenEdition}

Édition électronique

URL : http://journals.openedition.org/paleo/3111

DOI : 10.4000/paleo.3111

ISSN : 2101-0420

Éditeur

SAMRA

Édition imprimée

Date de publication : 30 décembre 2017

Pagination : 117-136

ISSN : $1145-3370$

\section{Référence électronique}

Marine Frouin, Christelle Lahaye, Norbert Mercier, Pierre Guibert, Isabelle Couchoud, Jean-Pierre Texier, Aurélien Royer, Sandrine Costamagno, Cédric Beauval, Laurence Bourguignon, Liliane Meignen, Jean-Guillaume Bordes, Guillaume Seguin, Hervé Bocherens, François Lacrampe-Cuyaubère, Xavier Muth, Eva-Maria Geigl, Rainer Grün, María Dolores Garralda, Bernard Vandermeersch, Alan E. Mann et Bruno Maureille, « Chronologie du site moustérien de type Quina des Pradelles (Marillac-le-Franc, Charente, France) », PALEO [En ligne], 28 | 2017, mis en ligne le 01 juin 2018, consulté le 07 juillet 2020. URL : http://journals.openedition.org/paleo/3111 ; DOI : https://doi.org/10.4000/paleo.3111

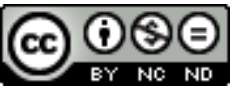

PALEO est mis à disposition selon les termes de la licence Creative Commons Attribution - Pas d'Utilisation Commerciale - Pas de Modification 4.0 International. 


\title{
Chronologie du site moustérien de type Quina des Pradelles (Marillac-le-Franc, Charente, France)
}

\author{
Marine FROUIN ${ }^{(a, b)}$, Christelle LAHAYE ${ }^{(b)}$, Norbert MERCIER ${ }^{(b)}$, \\ Pierre GUIBERT ${ }^{(b)}$, Isabelle COUCHOUD(c), Jean-Pierre TEXIER ${ }^{(d)}$, \\ Aurélien ROYER ${ }^{(e)}$, Sandrine COSTAMAGNO(f), Cédric BEAUVAL ${ }^{(9)}$, \\ Laurence BOURGUIGNON ${ }^{(h)}$, Liliane MEIGNEN ${ }^{(i)}$, \\ Jean-Guillaume BORDES ${ }^{(d)}$, Guillaume SEGUIN(j), Hervé BOCHERENS(k), \\ François LACRAMPE-CUYAUBÈRE ${ }^{(1, m)}$, Xavier MUTH ${ }^{(m)}$, Eva-Maria GEIGL ${ }^{(n)}$, \\ Rainer GRÜN( ${ }^{(0)}$, Maria Dolores GARRALDA ${ }^{(p)}$, \\ Bernard VANDERMEERSCH ${ }^{(d)}$, Alan E. MANN(q), Bruno MAUREILLE(d)
}

Résumé : Cet article présente une synthèse des données chronologiques du site des Pradelles (Marillac-le-Franc, Charente, France). Le gisement se caractérise dans son locus est par un remplissage de près de quatre mètres d'épaisseur ayant livré des dépôts de la fin du Paléolithique moyen dans lesquels une industrie moustérienne de type Quina est associée à une faune abondante largement dominée par le renne. Durant les fouilles conduites par B. Maureille et A. Mann entre 2001 et 2013, les données chronologiques obtenues pour ce locus ont été peu nombreuses : une datation par thermoluminescence (TL) sur un silex chauffé et une autre par U-Th sur un spéléothème situé dans la partie inférieure de la séquence. Des analyses par le radiocarbone ont été également réalisées sur des ossements provenant du sommet du remplissage, mais se sont révélées partiellement infructueuses en raison de l'ancienneté des vestiges proche de la limite de la méthode.

(a) Research Laboratory for Archaeology and the History of Art, University of Oxford, Dyson Perrins Building, South Parks Road, Oxford OX1 3QY, UK - marine.frouin@rlaha.ox.ac.uk

(b) CNRS, Univ. Bordeaux Montaigne, IRAMAT-CRP2A UMR 5060, FR-33607 Pessac - christelle.lahaye@u-bordeaux-montaigne.fr norbert.mercier@u-bordeaux-montaigne.fr-pierre.guibert@u-bordeaux-montaigne.fr

(c) CNRS, Univ. Savoie-Mont Blanc, MCC, EDYTEM UMR 5204, FR-73376 Le Bourget du lac cedex - isabelle.couchoud@univ-smb.fr (d) CNRS, Univ. Bordeaux, MCC, PACEA UMR 5199, FR-33615 Pessac cedex - j-pierre.texier@ wanadoo.fr ; jean-guillaume.bordes@u-bordeaux.fr ; vanderm13@gmail.com ; bruno.maureille@u-bordeaux.fr

(e) CNRS, Univ. Bourgogne, Biogéosciences UMR 6282, FR-21000 Dijon ; École Pratique des Hautes Études, Laboratoire EPHE PALEVO, FR-21000 Dijon - aurelien_royer@hotmail.com

(f) CNRS, Univ. Jean Jaurès Toulouse II, TRACES UMR 5608, FR-31058 Toulouse cedex - costamag@univ-tlse2.fr

(g) SARL Archéosphère, 2 rue des Noyers - FR-11500 Quirbajou - c.beauval @archeosphere.com

(h) INRAP Grand Sud-Ouest, Pôle mixte de Recherche de Campagne, FR-24260 Campagne - laurence.bourguignon@inrap.fr

(i) CNRS, Univ. Côte d'Azur, CEPAM UMR 7264, FR-06357 Nice - liliane.meignen@cepam.cnrs.fr

(j) Éveha Etude et valorisation archéologique, Route de Larnay, La Tardiverie, FR-86580 Biard - guillaume.seguin@eveha.fr

(k) Fachbereich Geowissenschaften, Forschungsbereich Paläobiologie - Biogeologie, Senckenberg Center for Human Evolution and Palaeoenvironment (HEP), Universität Tübingen, Hölderlinstr. 12, D-72074 Tübingen - herve.bocherens@uni-tuebingen.de

(I) SARL Archéosphère, 2 rue des Noyers, FR-11500 Quirbajou - Get in Situ Sarl, CH-Riex, Vaud - f.lacrampe@archeosphere.com (m) Get in Situ Sarl, CH-Riex, Vaud - x.muth@getinsitu.com

(n) Institut Jacques Monod, CNRS, Université Paris Diderot, UMR 7592, Équipe Épigénome et Paléogénome, 15 rue Hélène Brion, FR-75013 Paris - eva-maria.geigl@ijm.fr

(o) ARCHE, Environmental Futures Research Institute Griffith University, 170 Kessels Road, Nathan, QLD 4111, Australia Rainer.Grun@griffith.edu.au

(p) U. D. de Antropología Física. Facultad de Biología. Universidad Complutense de Madrid. E-28040 Madrid - mdgarral@ucm.es (q) Department of Anthropology, Princeton University, Princeton, NJ 08544, USA - mann@ princeton.edu 
Dans le cadre d'un projet pluridisciplinaire visant à établir des jalons chronologiques fiables pour l'évolution des cultures néandertaliennes du Sud-Ouest de la France, nous avons entrepris la datation des dépôts sédimentaires de la séquence des Pradelles par Luminescence Stimulée Optiquement (OSL). Les résultats de ces méthodes appliquées à différents minéraux (quartz et feldspaths), et combinés aux données radiométriques déjà disponibles, ont permis de préciser la chronostratigraphie du gisement. Ils permettent de situer chronologiquement les restes néandertaliens des Pradelles et les occupations Quina entre la fin du MIS (Marine Isotope Stage) 4 et pendant le MIS 3, jusqu'à 50 ka.

Mots-clefs : chronologie, Marillac, Les Pradelles, Neandertal, Quina, radiocarbone, OSL, TL, U-Th.

Abstract: Chronology of a Quina type mousterian site of Les Pradelles (Marillac-le-Franc, Charente, France). This paper presents a chronological framework for the site of Les Pradelles (Marillac-le-Franc, Charente, France). In the eastern part of the site, four meters of sediments preserve late Middle Paleolithic archaeological materials with Quina Mousterian lithics associated with abundant reindeer-dominated faunal remains. During the most recent excavations conducted by B. Maureille and A. Mann between 2001 et 2013, only a few dates were obtained in this part of the site: one was a single burnt flint dated by thermoluminescence (TL) and the second, was a single speleothem located at the base of the sequence dated using uranium-series disequilibrium (U-Th). Radiocarbon analysis were conducted on bones from the top layers but were partially unsuccessfull, since the results were close to the limit of the method.

As part of a multidisciplinary project that aims to define a reliable chronological framework for understanding the variability of Neandertal cultures in the SW France, we dated the sedimentary deposits of the Les Pradelles site using Optically Stimulated Luminescence (OSL) methods. The application of these techniques to various materials (quartz and feldspars), alongside previously obtained radiometric datasets, improve the chronostratigraphy of the site. They constrain the age of the Neandertal remains and the Quina occupations between the end of Marine Isotope Stage (MIS) 4 and during MIS 3, up to $~ 50$ ka BP.

Key-words: chronology, Marillac, Les Pradelles, Neandertal, Quina, radiocarbon, OSL, TL, U-Th.

\section{1- Introduction}

Le site des Pradelles (Marillac-le-Franc, Charente) aussi connu sous le nom de Marillac (Vandermeersch 1976) présente un remplissage sédimentaire rapporté à la fin du Paléolithique moyen dans lequel une industrie moustérienne de type Quina est associée à une faune abondante largement dominée par le renne (Meignen et Vandermeersch 1986 ; Meignen 1988 ; Bourguignon 1997 ; Maureille 2003 ; Maureille et al. 2010). Découvert en 1898, le gisement a dès lors été exploité en tant que carrière de pierres jusqu'au début de la seconde guerre mondiale. Dans les années 1930, P. Richeboeuf y découvre des vestiges paléolithiques, notamment un fragment de mandibule humaine. P. David y effectue quelques recherches très sommaires en 1934 et 1935 puis, après une période d'oubli, le site fait l'objet de fouilles entre 1967 et 1980 , conduites par B. Vandermeersch. Ces campagnes de fouilles ont permis la mise au jour de milliers de vestiges lithiques et fauniques, ainsi que celle d'une trentaine de restes de Néandertaliens, dont un arrière-crâne incomplet et une diaphyse fémorale d'enfant (Vandermeersch 1980 ; Garralda et al. 2014a, 2014b). Entre 2001 et 2013, les recherches reprirent sous la direction de deux d'entre nous (BM et AM) avec pour objectifs d'enrichir le corpus des vestiges archéologiques et paléoanthropologiques (Mussini 2011), de comprendre le fonctionnement anthropique du gisement, de mettre en évidence les processus de formation (Maureille et al. 2010), et d'en préciser la chronologie. Les premières datations absolues des
Pradelles suggèrent une mise en place des dépôts sédimentaires entre le stade isotopique 5 (par U-Th) et le stade isotopique 3 (par C-14) (Maureille et al. 2008). Ces datations, bien que peu nombreuses, constituent des jalons importants permettant d'esquisser une chronologie préliminaire de cette séquence, sans toutefois donner une attribution chronologique précise aux vestiges découverts.

Afin de compléter et d'affiner le cadre chronologique de la séquence des Pradelles, une étude portant sur l'application des méthodes de datation par Luminescence Stimulée Optiquement (Optically Stimulated Luminescence - OSL) a été conduite depuis 2011. Appliquée aux grains de quartz et de feldspaths contenus dans les sédiments et exposés à la lumière du jour avant leur enfouissement, l'OSL permet de déduire l'âge des dépôts. Les recherches menées ces 40 dernières années en datation par luminescence ont en effet montré que les quartz sont des dosimètres naturels fiables, de même que les feldspaths potassiques qui montrent généralement des signaux plus intenses mais dont la sensibilité à l'exposition à la lumière est différente des quartz. Dans cette étude, nous avons obtenu des données issues de signaux de luminescence aux caractéristiques différentes provenant de ces deux minéraux : signal OSL des quartz et signaux IRSL/pIRIRSL des feldspaths (cf. infra). La comparaison des résultats permet d'apprécier l'efficacité de la remise à zéro des minéraux dans le passé et ainsi, de s'assurer de la fiabilité des âges calculés. 
Cet article présente une synthèse des données chronologiques disponibles pour le gisement des Pradelles en intégrant les données numériques, anciennement $\left({ }^{14} \mathrm{C}\right.$, TL, U-Th) et nouvellement obtenues (OSL et IRSL/pIRIRSL) et les données paléoenvironnementales. L'implication de ces résultats sur la chronologie des sites paléolithiques moustériens du Sud-Ouest de la France et en particulier sur celle des occupations Quina peut ainsi être discutée.

\section{2 - Présentation du gisement des Pradelles (Marillac-Le-Franc)}

Le gisement des Pradelles est localisé sur la commune de Marillac-le-Franc en Charente, à une altitude de $108 \mathrm{~m}$. Sa limite jouxte le cours de la rivière Ligonne, affluent de la Tardoire. Le site des Pradelles a été probablement accessible pour les populations humaines et animales du Paléolithique suite à l'ouverture de chambres karstiques qui se sont développées dans les calcaires bajocienbathoniens. II se présente maintenant sous la forme d'une vaste dépression au sommet d'une très légère surélévation du relief (fig. 1). La dépression mesure aujourd'hui 19,7 mètres de long selon son axe ONO-ESE et 10,6 mètres selon l'axe NNE-SSO (largeur maximale). Elle est partiellement comblée par un puissant dépôt sédimentaire, fossilifère et archéologique pour la moitié est.

\section{1 - Contexte morphologique et géologique}

L'origine et l'histoire du site sont en partie liées à des phénomènes tectoniques qui régissent à plus grande échelle l'ensemble du « karst de La Rochefoucauld » dont il fait partie. Actuellement, le gisement est divisé en trois loci (fig. 1) : i) la partie est avec le dépôt sédimentaire le plus puissant qui a livré le plus grand nombre de vestiges, ii) la partie ouest qui contient un dépôt de plus faible puissance et iii) la « Grotte aux Poules » qui correspond à une petite galerie karstique située dans l'angle sud-ouest de la dépression. Cette dernière n'a livré aucun artefact lithique en place, il s'agit en fait d'un repaire d'hyènes des cavernes très partiellement conservé. Les analyses effectuées dans cette étude ont été focalisées sur les deux premiers loci.

Une première étude stratigraphique et sédimentologique des Pradelles a été réalisée par A. Debénath (1974), lors des fouilles dirigées par l'un d'entre nous (BV). Dans sa partie est, le remplissage atteint une épaisseur de près de $4 \mathrm{~m}$ qui fut subdivisée du haut vers le bas en 12 niveaux (Debénath 1974). À partir de 2001, l'étude réalisée par I. Couchoud et J.-P. Texier a mis en évidence de bas en haut la succession de 6 unités lithostratigraphiques (UL)

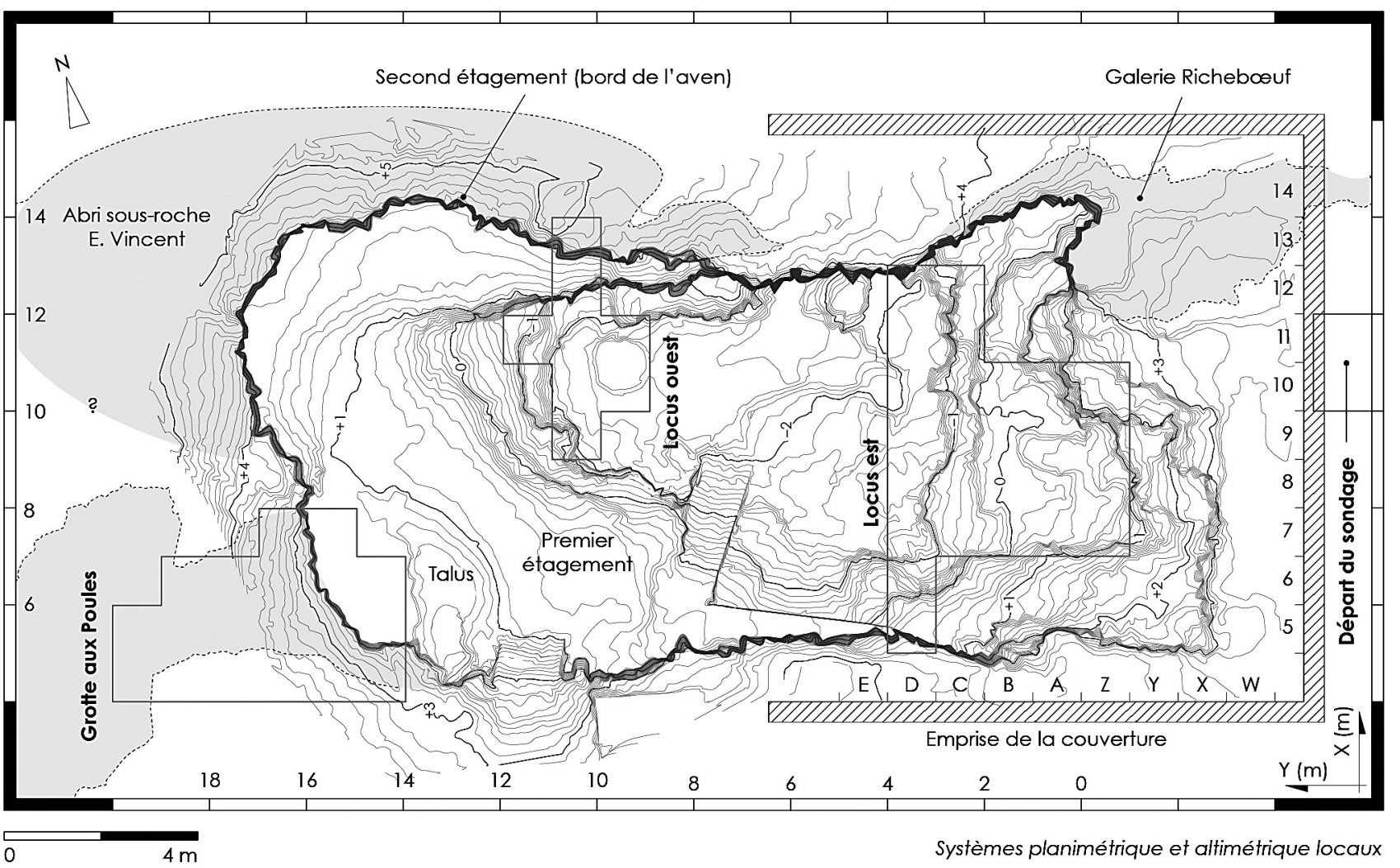

Figure 1 - Plan topographique des Pradelles et localisation des trois loci, levé et traitement topographique.

Figure 1 - Topographic map of Les Pradelles with the location of the three loci, topographic survey and treatment. 
comprenant 12 faciès (fig. 2 ; Texier et Couchoud 2007 ; Texier 2000) :

- UL A (faciès 1) : argiles limoneuses karstiques stériles. Celles-ci incluent localement un plancher stalagmitique qui a fait l'objet d'une datation par U-Th. II a été observé lors des fouilles dirigées par $B$. Vandermeersch près de l'interface entre ce substratum stérile et le remplissage sédimento-archéologique (Debénath in Maureille et al. 2010). Ce plancher est en fait inclus dans la partie supérieure de cette unité, recouvert par des argiles karstiques. En revanche, la relation stratigraphique de la base du plancher avec les argiles limoneuses de cette unité reste incertaine, il n'est donc pas exclu qu'il s'agisse d'un plancher suspendu, antérieur au remplissage argileux.

- UL B (faciès $2 a$ et $2 b$ ) : cailloutis plus ou moins colmatés par une matrice argilo-limoneuse brun foncé, riches en matériel archéologique et en coprolithes (pour le faciès $2 a$ ). - UL C (faciès $4 a, 4 b, 4 c, 4 d$ ) : fragments rocheux hétérométriques jointifs dont les vides d'entassement sont colmatés par une matrice à texture plus ou moins fine. Quatre faciès y ont été distingués sur la base de différences de couleur et de texture de la matrice. Cette unité contient un abondant matériel archéologique (surtout les faciès $4 \mathrm{a}$ et $4 b)$.

- UL D (faciès 5 et 6 ) : éboulis hétérométriques à structure ouverte à semi-ouverte (faciès 5). La partie sommitale de ces éboulis passe latéralement à des dépôts stratifiés (faciès 6). Le matériel archéologique est moins abondant dans le faciès 5 que les unités $B$ et $C$ et devient très rare dans le faciès 6 .

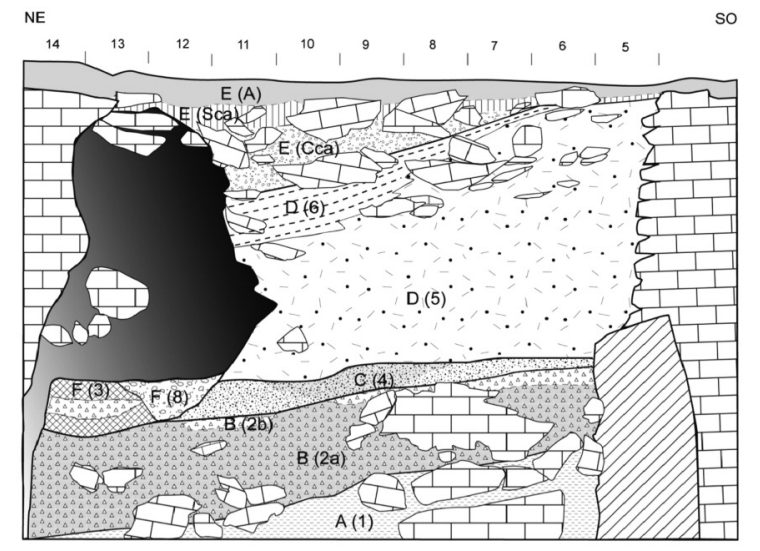

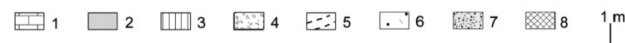

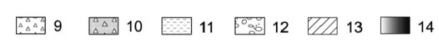

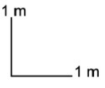

Figure 2 - Lithostratigraphie du locus est. Unités lithostratigraphiques (lettres) et faciès sédimentaires (numéros).

Figure 2 - Lithostratigraphy of the east locus. Lithostratigraphic units (letters) and sedimentary facies (numbers).
- UL E (faciès 7) : dépôt de sub-surface constitué de dalles calcaires et de cailloux très hétérométriques inclus dans une matrice sablo-argileuse brun rouge. Cette unité supporte un calcosol (Baize et Jabiol 1995).

- UL F (faciès 3 et 8 ) : dépôts colluviaux consécutifs à des remaniements sub-actuels.

Ces descriptions permettent de mieux comprendre les processus de mise en place des dépôts. L'UL A résulte d'écoulements fluviatiles de basse énergie en contexte endokarstique. Les UL B, C et D qui se superposent à celleci se sont formées après un effondrement du toit de la cavité et l'ouverture d'un aven. Ces unités proviennent pour l'essentiel d'une éboulisation des parois de la cavité, phénomène d'abord contemporain de débordements de la Ligonne (UL B), puis associé à des processus de ruissellement et de percolation en provenance du plateau (UL C et D). Ces derniers ont été importants lors de la mise en place de l'UL $C$, puis très peu actifs lors de la sédimentation de l'UL D. Lors du dépôt de la partie sommitale de l'UL $D$ se sont manifestés des phénomènes de solifluxion responsables de la formation du faciès stratifié 6. L'UL E témoigne de l'effondrement d'un plafond résiduel et l'UL F est reliée à l'exploitation du site en carrière antérieurement aux différentes fouilles pratiquées.

Les corrélations stratigraphiques entre les loci est et ouest sont difficiles à établir à cause d'un hiatus sédimentaire entre ces deux parties du gisement. Dans la partie ouest, trois unités (voir fig. 4B) ont été identifiées, de bas en haut (Maureille et al. 2008) :

- Unité 1 : dépôts argilo-limoneux possédant les caractéristiques de l'UL A de la partie est.

- Unité 2a' : diamicton à support clastique constitué de fragments calcaires et siliceux inclus dans une matrice argilo-limoneuse de couleur brun rouge. Certains débris rocheux, fortement altérés, sont réduits à l'état de « fantômes ».

- Unité 3 : dépôts de remaniement.

L'unité 1 correspond à la même phase sédimentaire de dépôts fluviatiles endokarstiques que l'UL A de la partie est (Texier et Couchoud 2007). L'unité 2a' est le résultat à la fois du remaniement de l'unité 1 lié à l'activité de l'hyène des cavernes et de l'éboulisation des parois de la cavité. Elle pourrait être contemporaine de l'UL E observée dans la partie est. L'unité 3 correspond à des sédiments remaniés y compris depuis la fin des fouilles de 1965 à 1980. Dans cette partie du site, il existerait donc un hiatus temporel important entre les différentes unités identifiées.

\section{2 - La présence humaine aux Pradelles}

\subsection{1 - Les industries}

Les études technologiques et typologiques du matériel lithique provenant de l'UL $B$ (faciès $2 a$ et $2 b$ ) des fouilles de $\mathrm{BM}$ et $\mathrm{AM}$ (correspondant aux couches 11 à 9 des fouilles dirigées par BV, Maureille et al. 2008) ont permis de rapporter ces vestiges au technocomplexe lithique Quina (Meignen et Vandermeersch 1986 ; Meignen 1988 ; 
Bourguignon 1996, 1997) dont il constitue actuellement l'un des assemblages de référence. L'étude des caractéristiques de l'outillage et de l'économie des matières premières de ces deux couches (Meignen et Vandermeersch, ibidem ; Meignen 1988 ; Meignen et al. $2007,2010)$ met en évidence une gestion différentielle des matières premières en fonction de leur qualité technique et des outils recherchés. Les silex locaux, de mauvaise qualité, sont débités sur place et servent à la réalisation de racloirs, encoches et denticulés. Les silex non locaux de bonne qualité, dont les gîtes seraient situés à 1520 kilomètres du site, constituent la matière première préférentiellement utilisée pour la confection des racloirs Quina. Ces silex allochtones ont été généralement introduits dans le site directement sous la forme d'outils. Ils ont ensuite été très souvent réavivés, voire recyclés, puis abandonnés sur place (Bourguignon 2001 ; Bourguignon et al. 2013). Quelques galets ou fragments de galets en quartz provenant d'alluvions ont été utilisés comme percuteurs (Maureille et al. 2010). Dans cette unité, l'un des éléments marquants des occupations humaines est la très faible densité de matériel lithique en comparaison avec l'abondance des restes osseux (Costamagno et al. 2005, 2006).

\subsection{2 - La faune chassée}

L'UL B (faciès $2 a$ et $2 b$ ) est très riche en matériel faunique. L'abondance décroît progressivement dans l'UL C (faciès $4 a, 4 b$ et $4 d$ ), puis dans l'UL D (faciès 5 ). Pour l'ensemble de la séquence, le renne est le taxon dominant (à $90 \%$ ), suivi du bison, du cheval ainsi que la marmotte dans les faciès supérieurs (faciès $4 \mathrm{~b}$ et 5 ) de la stratigraphie (Maureille et al. 2008, 2010, 2013). L'hypothèse la plus prudente pour expliquer l'accumulation des vestiges fauniques des faciès $2 a, 2 b$ et $4 a$ est que le site des Pradelles ait fonctionné comme une halte de chasse. Elle se caractérise alors par une exploitation extrême et performante de toutes les ressources disponibles sur les ongulés (peau, tendon, viande et moelle ; Costamagno et al. 2006). Les carcasses semblent y avoir été introduites après qu'un premier traitement ait été conduit sur le site d'abattage, où les parties pauvres auraient été abandonnées. Seuls les éléments riches en graisse et moelle ont été apportés pour y être récupérés et consommés sur place ou exportés pour une consommation différée. L'exploitation intense des carcasses animales se matérialise également par la récupération de très nombreuses diaphyses pour leur utilisation en retouchoirs (plus 700 retouchoirs, Costamagno et al. 2017).

On peut noter aussi la présence discrète de carnivores troglophiles comme l'hyène des cavernes, identifiée par la découverte de ses coprolithes en fragments dans tous les faciès et intacts à la base du faciès $2 a$. Un changement progressif de l'impact de prédateurs sur l'assemblage faunique a été identifié (Costamagno et al. 2005). L'UL B (faciès $2 a$ et $2 b$ ) localisée en bas de séquence et l'UL $C$ (faciès $4 a$ ) présentent des caractéristiques très proches : l'abondance des traces anthropiques et la rareté des traces de carnivores présentes majoritairement sur des fragments diaphysaires indiquent que les animaux ont été chassés par les néandertaliens puis secondairement charognés par les carnivores (Costamagno et al. ibid.). Au sommet de la séquence dans les unités UL $C$ (faciès $4 b$ ) et UL $D$ (faciès 5), l'impact des carnivores est plus important mais la présence d'ossements avec des traces anthropiques indiquerait toujours une origine mixte des ossements (Costamagno et al. 2005).

\subsection{3 - Les restes humains}

Depuis 1934, 87 pièces humaines ont été mises au jour aux Pradelles dans les UL B et $\mathrm{C}$, correspondant à un minimum de sept individus se rapportant à la lignée néandertalienne : trois adultes, deux adolescents et deux enfants (Maureille et al. 2010). D'après Mussini et al. (2010), il apparaît que les Néandertaliens ont appliqué un traitement particulier à leurs morts. En effet, seules certaines parties du squelette ont été découvertes dans le site, à savoir le crâne et les os des membres. Ces parties du corps ont été écorchées et décharnées et leurs vestiges osseux présentent un grand nombre de stries de découpe, de traces de fracturation sur os frais, des points d'impacts, et la probable récupération des extrémités articulaires (Mussini 2011). L'hypothèse avancée pour expliquer ce comportement est celle du cannibalisme à but alimentaire (Boulestin 2006), puisqu'il est proche du traitement appliqué à la faune sur ce site (Mussini 2011) mais d'autres interprétations n'entrant pas dans la sphère économique peuvent être envisagées par certains d'entre nous (Garralda 2008 ; Garralda et al. 2005, 2014a, 2014b). Enfin, certains vestiges abandonnés par les vivants ont été ensuite charognés par des carnivores modifiant ainsi leur morphologie initiale (Maureille et al. 2017 ce volume) et impactant donc l'intégrité de l'assemblage des restes humains.

\section{3 - Données géochronologiques}

\section{1 - Datation des ossements par radiocarbone AMS}

Les études isotopiques sur ossements fauniques et humains ont montré la bonne conservation du collagène dans la majorité des spécimens testés (Bocherens et al. 1991, 2005 ; Fizet et al. 1995), permettant d'envisager l'application de la datation radiocarbone par AMS sur ossements (Maureille et al. 2008). En 2004, trois fragments osseux provenant de la partie sommitale de la stratigraphie de la partie est ont été sélectionnés : i) un troisième métatarsien droit d'Ursus spelaeus (Beta - 191680) provenant du faciès $7 / 8$ (UL E/F) ; ii) un fragment d'os long épais de bovidé ou d'équidé (Beta - 191681) provenant du faciès 6 (UL D) ; iii) un fragment de métacarpien de renne provenant du faciès 5 (UL D) et portant de nombreuses traces de découpes (Beta - 191679).

L'analyse des échantillons a été effectuée par le laboratoire Beta Analytic Inc (Miami, USA) en appliquant une méthode classique de décontamination des ossements de type AAA (Acide Alcalin Acide). Les âges ont été calibrés à l'aide du 


\begin{tabular}{|c|c|c|c|c|c|c|c|c|c|c|}
\hline Echantillon & Locus & $\begin{array}{l}\text { UL (faciès } \\
\text { ou unité) }\end{array}$ & $\begin{array}{c}\text { Référence } \\
\text { Beta Analytic }\end{array}$ & Prétrait. & $\begin{array}{c}\text { Poids initial } \\
\text { de } \\
\text { l'échantillon } \\
(\mathrm{g})\end{array}$ & $\begin{array}{c}\text { Collagène } \\
\text { extrait } \\
\text { (mg) }\end{array}$ & $\begin{array}{c}\% \% \\
\text { combustion } \\
\text { du } \\
\text { carbone }\end{array}$ & $\begin{array}{c}{ }^{13} \mathrm{C} / /^{12} \mathrm{C} \\
\text { ratio } \\
\text { o/oo }\end{array}$ & $\begin{array}{c}\operatorname{Age} \pm 1 \sigma \\
\left(\mathrm{BP}^{\mathrm{a}}\right)\end{array}$ & $\begin{array}{l}\text { Age calibré } \\
\text { (cal BP) } \\
95,4 \% \text { de } \\
\text { probabilité }\end{array}$ \\
\hline $\begin{array}{c}\text { 3ème métacarpien } \\
\text { d'ours des cavernes }\end{array}$ & Est & E/F (7/8) & 191680 & $\begin{array}{l}\text { Acide } \\
\text { alcalin }\end{array}$ & 5,3 & 135,1 & 40,7 & $-17,7$ & $32440 \pm 440$ & $37900-35430$ \\
\hline $\begin{array}{l}\text { Fragment os long } \\
\text { bovidé / équidé }\end{array}$ & Est & $\mathrm{D}(6)$ & 191681 & $\begin{array}{l}\text { Acide } \\
\text { alcalin }\end{array}$ & 10,7 & 181,4 & 39,1 & $-20,6$ & $>46780$ & - \\
\hline $\begin{array}{l}\text { Métacarpien de renne } \\
\text { avec traces de } \\
\text { découpe }\end{array}$ & Est & $\mathrm{D}(5)$ & 191679 & $\begin{array}{l}\text { Acide } \\
\text { alcalin }\end{array}$ & 3,2 & 226,3 & 35,2 & $-20,2$ & $>45240$ & - \\
\hline Métatarsien de cheval & Ouest & (2a') & 219943 & $\begin{array}{l}\text { Acide } \\
\text { alcalin }\end{array}$ & 4,9 & 105,5 & 43,3 & $-21,3$ & $40660 \pm 530$ & $45200-43260$ \\
\hline $\begin{array}{c}\text { Fragment d'humérus } \\
\text { de renne }\end{array}$ & Ouest & (2a') & 219945 & $\begin{array}{l}\text { Acide } \\
\text { alcalin }\end{array}$ & 5,6 & 121,7 & 36,0 & $-19,5$ & $>42000$ & - \\
\hline $\begin{array}{l}\text { Fragment d'humérus } \\
\text { d'hyène des cavernes }\end{array}$ & $\begin{array}{l}\text { Grotte } \\
\text { aux } \\
\text { poules }\end{array}$ & $\begin{array}{l}\text { Remanié } \\
\text { Int. }\end{array}$ & 219942 & $\begin{array}{l}\text { Acide } \\
\text { alcalin }\end{array}$ & 4,0 & 8,1 & 41,7 & $-19,3$ & $41860 \pm 1140$ & $48020-43330$ \\
\hline $\begin{array}{c}\text { Métacarpien de Bos / } \\
\text { Bison }\end{array}$ & $\begin{array}{l}\text { Grotte } \\
\text { aux } \\
\text { poules }\end{array}$ & $\begin{array}{l}\text { Remanié } \\
\text { Int. }\end{array}$ & 219944 & $\begin{array}{l}\text { Acide } \\
\text { alcalin }\end{array}$ & 4,0 & 24,6 & 36,0 & $-20,9$ & $39930 \pm 490$ & $44530-42820$ \\
\hline
\end{tabular}

${ }^{\mathrm{a}} \mathrm{BP}=$ Before Present de 1950

${ }^{\mathbf{b}}$ Les âges ont été calibrés à l'aide du logiciel Oxcal 4.3 (Bronk Ramsey, 2009 ; mise à jour: 11/11/2017) et de la courbe de calibration IntCal13 (Reimer, 2013).

${ }^{\mathrm{a}} \mathrm{BP}=$ Before Present (i.e. 1950).

${ }^{b}$ Ages have been calibrated using Oxcal 4.3 (Bronk Ramsey, 2009 ; last updated: 11/11/2017) and from the calibration curve IntCal13 (Reimer, 2013)

Tableau 1 - Données relatives aux datations par radiocarbone des Pradelles.

Table 1 - Radiocarbon dates from Les Pradelles.

logiciel Oxcal 4.2 (Bronk Ramsey 2009) utilisant la courbe de calibration IntCal13 (Reimer 2013). Les résultats détaillés ici sont fournis dans le tableau 1. Pour l'UL D, Les âges des vestiges sont supérieurs à 45000 ans BP. La pièce provenant du faciès $7 / 8$ (UL E/F) a donné un âge compris entre 37900 et 35430 ans cal BP (niveau de confiance à 95,4 \%, 2 $\sigma$ ). Les résultats permettent de proposer que la fin du comblement sédimentaire de l'aven s'est produite à la fin du stade isotopique 3. Cet âge pour I'UL E/F constitue donc un Terminus Ante Quem de l'occupation du site.

Le locus ouest des Pradelles a également fait l'objet de deux datations par le radiocarbone (par le même laboratoire) sur deux échantillons provenant de l'unité $2 a^{\prime}$ (tabl. 1). Les résultats indiquent une occupation comprise entre 45200 et 43260 ans cal BP (2б) pour le premier échantillon, alors que la seconde pièce a fourni un âge supérieur à 42000 ans BP. Ces âges suggèrent que l'occupation animale du locus ouest pourrait être corrélée avec celle de la partie sommitale de l'UL D. Enfin, deux autres datations numériques (tabl. 1) ont aussi été obtenues pour la Grotte aux Poules et semblent placer son occupation par l'hyène des cavernes à une période contemporaine des occupations humaines du locus ouest, entre 44800 et 43120 ans cal BP $(2 \sigma ; n=2$; fonction $R \_$Combine).

\section{2 - Datation d'un silex chauffé par thermoluminescence}

Une datation par thermoluminescence a été réalisée par P. Guibert et E. Vieillevigne en 2006, sur un silex brûlé
(BDX 10727) sélectionné parmi les vestiges lithiques des Pradelles. II s'agit d'un grattoir en matériau allochtone provenant du faciès $2 b$ (UL B). Le silex a été broyé mécaniquement puis la fraction granulométrique comprise entre 80 et $200 \mu \mathrm{m}$ a été traitée à l'acide chlorhydrique $\mathrm{HCl}$ $1 \mathrm{M}$ afin de dissoudre d'éventuels carbonates, puis par une solution $(\mathrm{HCl} 1 \mathrm{M}, \mathrm{HF} 0,5 \mathrm{M})$ afin d'éliminer la partie superficielle des grains et ainsi éviter des signaux parasites dus au broyage.

Pour les mesures de TL, les grains de fraction granulométrique 80-200 $\mu \mathrm{m}$ ont été déposés dans des nacelles de laiton (aliquotes) à l'aide d'un répartiteur volumétrique. La dose équivalente a été déterminée suivant un protocole de "doses ajoutées en aliquotes multiples", destiné à construire une courbe de croissance de l'intensité du signal TL en fonction de la dose naturelle et de la dose d'irradiation beta ajoutée (fig 3a). Comme le prévoit aussi le protocole, une deuxième courbe de croissance a été construite à partir de signaux induits par des doses régénérées sur des grains préalablement vidés de leur signal naturel (remis à zéro thermiquement une heure à $450^{\circ} \mathrm{C}$ ). La paléodose est déterminée à partir des courbes de thermoluminescence de première lecture dont la croissance avec la dose varie selon la fonction définie par les courbes de seconde lecture (fig. 3b). L'intervalle de température pour lequel la paléodose est calculée a été préalablement déterminé à partir du test du plateau, qui permet de ne sélectionner que la zone de températures correspondant aux pièges les plus stables, pour lesquelles la dose bêta équivalente calculée est constante. Pour l'échantillon des Pradelles, ce domaine est compris entre 350 et $480^{\circ} \mathrm{C}$ (fig. 3b). La dose équivalente bêta $(31,1 \pm$ 

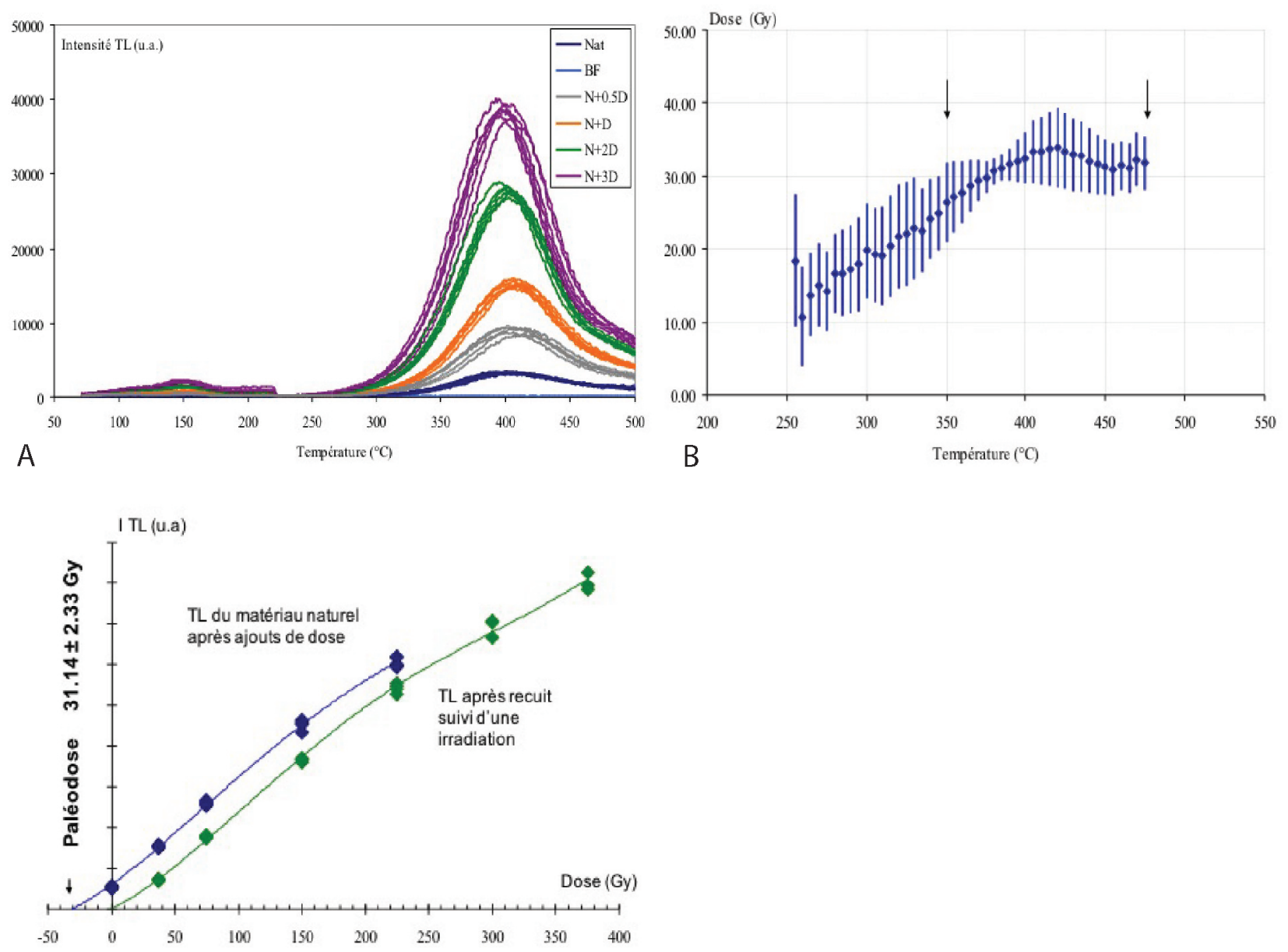

C

Figure 3 - A) Courbes de première lecture du silex BDX 10727. Le chauffage a été réalisé sous atmosphère d'azote à la vitesse de $4^{\circ} \mathrm{C} / \mathrm{s}$, avec un palier à $220^{\circ} \mathrm{C}$ pendant $2 \mathrm{~min}$. B) Test du plateau de BDX 10727. La dose équivalente bêta a été déterminée à partir des courbes $T L$ de 1ère et 2ème lectures, par approximation polynomiale. Les barres d'erreur représentent l'incertitude statistique. Cet échantillon présente un plateau large. Le domaine d'intégration a été choisi entre 350 et $480^{\circ} \mathrm{C}$. C) Loi d'acquisition de BDX 10727 et détermination de la dose équivalente bêta. Les points de $2^{\circ}$ lecture permettent de déterminer une loi de croissance de la TL, approchée de manière satisfaisante par un polynôme de degré 3 passant par l'origine (Guibert et al. 1996).

Figure 3 - A) TL glow curves for the BDX 10727 flint sample. The heating was carried out under a nitrogen atmosphere at the rate of $4^{\circ} \mathrm{C} / \mathrm{s}$, with a step at $220^{\circ} \mathrm{C}$ for 2 minutes. B) Plateau test for the BDX 10727 sample. The beta equivalent dose was determined from the $T L$ curves of the 1 st and 2 nd readings, by polynomial approximation. The error bars represent statistical uncertainty. This sample has a wide plateau. The integration range was chosen between 350 and $480^{\circ} \mathrm{C}$. C) Acquisition and determination of the beta equivalent dose. The second reading points make it possible to determine the TL growth curve, approximated by a polynomial of degree 3 passing through the origin (Guibert et al. 1996).

2,3 Gy ; notée paléodose sur la fig. 3c) a alors été déterminée à partir de la loi d'acquisition de la TL.

Le facteur d'efficacité des particules alpha, noté $k$, a été déterminé pour ce silex en comparant l'intensité des signaux de TL générés par une irradiation alpha à celle générée par des irradiations bêta. Pour cet échantillon, le coefficient d'efficacité des particules alpha k est de 0,057 \pm 0,011 .

Pour un silex archéologique, dont les dimensions sont typiquement centimétriques, la dose annuelle d'irradiation est en partie issue de l'échantillon lui-même, et en partie issue de son environnement. La dose d'irradiation alpha et bêta due à chacune de ces deux composantes a été calculée à partir des teneurs en ${ }^{40} \mathrm{~K},{ }^{235} \mathrm{U},{ }^{238} \mathrm{U}$, et ${ }^{232} \mathrm{Th}$ et de leurs descendants mesurés en laboratoire par spectrométrie gamma (tabl. 2) à bas bruit de fond et haute résolution (Guibert et Schvoerer 1991). La fraction de dose bêta provenant de l'environnement a été calculée suivant les recommandations d'Aitken (1985). Pour déterminer la dose annuelle gamma, nous avons dû reconstruire l'environnement dosimétrique de l'unique silex qui s'est avéré datable par TL au cours de cette étude (Guibert et al. 1998). En effet, celui-ci a été mis au jour dans un contexte hétérogène, composé de sédiments fins et d'éléments plus grossiers de natures et de radioactivités différentes. Nous 
avons caractérisé chaque classe d'éléments lithiques composant les niveaux $2 b$ (UL B), d'où est issu le silex, et 4a (UL C), qui le recouvre, puis, connaissant la position à laquelle l'échantillon a été recueilli, nous avons déterminé que la contribution effective du niveau $4 a$ représentait environ $8 \%$ de la dose dose reçue et celle des deux souscouches 2b (moyennées) $92 \%$. Nous avons pu ainsi calculer la composante gamma moyenne de la dose annuelle due aux sédiments environnant le silex. La composante due aux rayons cosmiques a été calculée en fonction de la profondeur d'enfouissement de l'échantillon (Prescott et Hutton 1994).

L'ensemble de ces résultats est présenté dans le tableau 2. Les analyses de TL et de dosimétrie associée ont permis

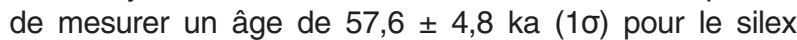
chauffé, ce qui correspond à la fin du MIS (Marine Isotope Stage) 4 ou au début du MIS 3 (d'après la chronostratigraphie de Lisiecki et Raymo 2005).

\section{3 - Datation par U-Th d'un plancher stalagmitique situé à la base du remplissage}

Un échantillon du plancher stalagmitique inclus dans la partie sommitale des argiles limoneuses de l'UL A (faciès 1) du locus est a été daté en 2007 par la méthode U-Th par MC-ICPMS (multi-collector inductively coupled plasma mass spectrometry) au Bristol Isotope Group facilities (University of Bristol, UK) par I. Couchoud avec la collaboration de D. Hoffmann.
L'échantillon a d'abord été dissous puis un spike composé de ${ }^{229} \mathrm{Th} / 236 \mathrm{U}$ a été ajouté à la solution avant de le verser dans une colonne d'échanges ioniques. Les fractions d'uranium et de thorium ont été séparées en utilisant des techniques classiques d'échange d'anions sur résines. Les mesures isotopiques des isotopes de la série de l'uranium ont été réalisées sur un MC-ICPMS ThermoFinnigan Neptune. De plus amples détails sur la préparation chimique des échantillons et les procédures de mesure par MC-ICPMS sont donnés dans Hoffmann et al. (2007) et Hoffmann (2008). Pour compenser la contribution de 230Th${ }^{234} U-238 U$ terrigène qui pourrait vieillir artificiellement l'âge de l'échantillon, une correction a été réalisée en appliquant la valeur de 0,8 $\pm 0,4$ (valeur globale de la croûte supérieure de la Terre, d'après Wedepohl 1995) pour le rapport d'activité $238 \mathrm{U} / 232 \mathrm{Th}$ de la fraction détritique et en supposant qu'elle ait atteint l'équilibre séculaire (tabl. 3). Toutefois, cette correction n'est qu'une hypothèse puisque le véritable rapport d'activité $238 \mathrm{U} / 232 \mathrm{Th}$ dans ce spéléothème en particulier peut être différent de la valeur moyenne de la croûte terrestre (e.g. Richards and Dorale 2003).

Les valeurs non corrigées donnent un âge de $83,15 \pm 0,52$ ka $(2 \sigma)$ et, après correction détritique, un âge de 81,96 \pm

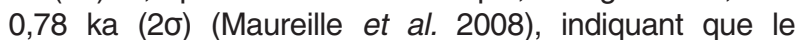
spéléothème s'est formé au cours du MIS 5a. Cette date constitue donc un Terminus Post Quem de l'occupation animale et humaine du site des Pradelles, qui à ce momentlà n'était pas encore ouvert sur l'extérieur.

\begin{tabular}{|c|c|c|c|c|c|c|c|c|c|c|c|c|c|c|}
\hline \multirow{3}{*}{ Faciès } & \multirow{3}{*}{ Echantillon } & \multicolumn{4}{|c|}{ Radioéléments ${ }^{\mathrm{a}}$} & \multicolumn{6}{|c|}{ Contribution à la dose annuelle (mGy/an éq $\beta$ ) } & \multirow{3}{*}{$\begin{array}{c}D_{a} \\
(\mathbf{G y} / \mathbf{k a})\end{array}$} & \multirow{3}{*}{$\begin{array}{c}D_{e} \\
(\text { Gy) }\end{array}$} & \multirow{3}{*}{$\begin{array}{l}\text { Age } \\
\text { (ka) }\end{array}$} \\
\hline & & \multirow{2}{*}{$\begin{array}{c}\mathrm{K} \\
(\%)\end{array}$} & \multirow{2}{*}{$\begin{array}{c}\mathbf{U}_{\text {tête }} \\
\text { (eq.ppm) }\end{array}$} & \multirow{2}{*}{$\underset{\text { (eq.ppm) }}{\mathbf{U}_{\text {fin }}}$} & \multirow{2}{*}{$\begin{array}{c}\text { Th } \\
(\mathbf{p p m})\end{array}$} & \multicolumn{2}{|c|}{ interne $^{b}$} & \multicolumn{3}{|c|}{ externe } & \multirow[t]{2}{*}{ Total } & & & \\
\hline & & & & & & bêta & alpha & bêta & gamma & cosm. & & & & \\
\hline $2 b$ & $\begin{array}{c}\text { Silex BDX } \\
10727\end{array}$ & $\begin{array}{c}0,03 \pm \\
0,01\end{array}$ & $\begin{array}{c}0,39 \pm \\
0,11\end{array}$ & $\begin{array}{c}0,27 \pm \\
0,02\end{array}$ & $\begin{array}{c}0,11 \pm \\
0,05\end{array}$ & $\begin{array}{c}0,06 \pm \\
0,01\end{array}$ & $\begin{array}{c}0,047 \pm \\
0,010\end{array}$ & $\begin{array}{c}0,10 \pm \\
0,01\end{array}$ & $\begin{array}{c}0,24 \pm \\
0,01\end{array}$ & $\begin{array}{c}0,09 \pm \\
0,01\end{array}$ & $\begin{array}{c}0,54 \pm \\
0,02\end{array}$ & $\begin{array}{c}0,54 \pm \\
0,02\end{array}$ & $\begin{array}{l}31,1 \\
\pm 2,3\end{array}$ & $\begin{array}{c}57,6 \pm \\
4,8\end{array}$ \\
\hline $\begin{array}{c}2 \mathrm{~b} \\
\left(1^{\text {er }} \text { sous- }\right. \\
\text { couche })\end{array}$ & sédiment & $\begin{array}{c}0,24 \pm \\
0,01\end{array}$ & $\begin{array}{c}1,16 \pm \\
0,06\end{array}$ & $\begin{array}{c}0,98 \pm \\
0,01\end{array}$ & $\begin{array}{c}2,11 \pm \\
0,03\end{array}$ & & & & & & & & & \\
\hline $\begin{array}{c}2 \mathrm{~b} \\
\left(2^{\text {nd }} \text { sous- }\right. \\
\text { couche })\end{array}$ & sédiment & $\begin{array}{c}0,30 \pm \\
0,01\end{array}$ & $\begin{array}{c}1,35 \pm \\
0,06\end{array}$ & $\begin{array}{c}1,08 \pm \\
0,01\end{array}$ & $\begin{array}{c}2,54 \pm \\
0,03\end{array}$ & & & & & & & & & \\
\hline $4 a$ & sédiment & $\begin{array}{c}0,29 \pm \\
0,01\end{array}$ & $\begin{array}{c}1,50 \pm \\
0,06\end{array}$ & $\begin{array}{c}1,01 \pm \\
0,01\end{array}$ & $\begin{array}{c}2,86 \pm \\
0,03\end{array}$ & & & & & & & & & \\
\hline
\end{tabular}

${ }^{\mathrm{a}}$ Les incertitudes représentent un écart-type de comptage. La teneur en $\mathrm{U}_{\text {tête }}$ est mesurée à partir des émissions $\gamma$ de ${ }^{235} \mathrm{U}$ et ${ }^{234} \mathrm{Th}$. La teneur $\mathrm{U}_{\text {fin }} \mathrm{a}$ été mesurée à partir des raies de ${ }^{214} \mathrm{~Pb}$ et ${ }^{214} \mathrm{Bi}$ en supposant l'équilibre entre ${ }^{226} \mathrm{Ra}$ et ${ }^{222} \mathrm{Rn}$ (ppm : partie par million).

${ }^{\mathrm{b}}$ le coefficient d'efficacité alpha, $\mathrm{k}$, vaut 0,057 $\pm 0,011$ et la dose interne gamma du silex est de 0,0007 $\pm 0,0001 \mathrm{mGy} / \mathrm{an}$.

\footnotetext{
${ }^{a}$ The uncertainties are given at one standard deviation. The $U_{\text {top }}$ content is measured from the $\gamma$ emissions of the ${ }^{235} U$ and ${ }^{234} \mathrm{Th}$. The $\mathrm{U}_{\text {bottom }}$ content has been measured from ${ }^{214} \mathrm{~Pb}$ and ${ }^{214} \mathrm{Bi}$ emission rays, assuming the equilibrium between ${ }^{226} \mathrm{Ra}$ and ${ }^{222} \mathrm{Rn}$ (ppm : part per million). ${ }^{\mathrm{b}}$ The Alpha efficiency coefficient, $\mathrm{k}$, is $0.057 \pm 0.011$ and the internal gamma dose of the flint is $0.0007 \pm 0.0001 \mathrm{mGy} / \mathrm{an}$.
}

Tableau 2 - Composition radiochimique, résultat de l'étude de dose annuelle reçue et âge du silex BDX 10727 des Pradelles. Pour les sédiments des niveaux $2 b$ et $4 a$, les teneurs équivalentes moyennes sont données en prenant en compte la composition en sédiment fin et en éléments lithiques (calcaires et autres roches).

Table 2 - Radioisotopic composition, results of the analysis of the annual dose and age are given for the flint sample BDX 10727 of Les Pradelles. For the sediments in the facies $2 b$ and $4 a$, there are equivalent average contents are given considering the proportion of fine sediment and lithic material (calcareous and other stones). 


\begin{tabular}{|c|c|c|c|r|r|r|r|c|}
\hline $\begin{array}{c}\text { Code échantillon } \\
\text { (Bristol Isotope Group) }\end{array}$ & $\begin{array}{c}{ }^{238} \mathbf{U} \\
(\mathrm{ng} / \mathrm{g})\end{array}$ & $\begin{array}{c}{ }^{232} \mathbf{T h} \\
(\mathrm{ng} / \mathrm{g})\end{array}$ & $\begin{array}{c}{ }^{230} \mathbf{T h} \\
(\mathrm{pg} / \mathrm{g})\end{array}$ & $\left.{ }^{230} \mathbf{T h} /{ }^{232} \mathbf{T h}\right)_{\mathbf{A}}$ & $\left(^{230} \mathbf{T h} /{ }^{238} \mathbf{U}\right)_{\mathbf{A}}$ & $\left(^{234} \mathbf{U} /{ }^{238} \mathbf{U}\right)_{\mathbf{A}}$ & $\begin{array}{c}\text { Age non corrigé } \\
(\mathrm{ka} / 2007)\end{array}$ & $\begin{array}{c}\text { Age corrigé } \\
(\mathrm{ka} / 2007)\end{array}$ \\
\hline BIG-UTh-L-4 & 244,928 & 10,473 & 2,1971 & 39,167 & 0,5480 & 1,0254 & $83,15 \pm 0,53$ & $81,96 \pm 0,78$ \\
\hline
\end{tabular}

Les erreurs analytiques sont données à $2 \sigma$ ( $95 \%$ de niveau de confiance).

« A » en indice signifie « rapport d'activité ».

Les constantes de désintégration utilisées sont $9,1577 \times 10^{-6} \mathrm{a}^{-1}$ pour ${ }^{230} \mathrm{Th}, 2,826 \times 10^{-6} \mathrm{a}^{-1}$ pour ${ }^{234} \mathrm{U}$, et $1,55125 \times 10^{-10} \mathrm{a}^{-1}$ pour ${ }^{238} \mathrm{U}$.

L'âge corrigé est calculé en utilisant un rapport d'activité initiale $\left({ }^{238} \mathrm{U} /{ }^{232} \mathrm{Th}\right)_{\mathrm{A} 0}=0,8 \pm 0,4$

L'année de référence est celle de la préparation chimique de l'échantillon (2007).

Analytical errors are given at $2 \sigma(95 \%$ level of confidence).

"A » means « activity ratio ».

The decay constants are $9.1577 \times 10^{-6} \mathrm{a}^{-1}$ for ${ }^{230} \mathrm{Th}, 2.826 \times 10^{-6} \mathrm{a}^{-1}$ for ${ }^{234} \mathrm{U}$, and $1.55125 \times 10^{-10} \mathrm{a}^{-1}$ for ${ }^{238} \mathrm{U}$.

The corrected age is calculated using the inital activity ratio $\left({ }^{238} \mathrm{U} /{ }^{232} \mathrm{Th}\right)_{\mathrm{A} 0}=0.8 \pm 0.4$

The reference year is the year of the sample chemical preparation (2007).

Tableau 3 - Résultats de la datation U-Th par MC-ICPMS de l'échantillon de plancher stalagmitique localisé à la base du remplissage des Pradelles (UL A, faciès 1).

Table 3 - Summary of the data involved in the U-Th dating by MC-ICPMS of the speleothem sample localised at the base of the deposit of Les Pradelles (UL A, facies 1).

\section{4 - Datation des sédiments par luminescence stimulée optiquement}

En novembre 2011 et juillet 2012, dix échantillons de sédiment ont été prélevés dans les loci est et ouest en vue de datations par OSL (fig. 4). Après nettoyage des coupes sur une profondeur de cinq centimètres, les échantillons ont été récoltés par grattage dans des sacs plastiques opaques sous un abri artificiel réalisé pour l'occasion avec une bâche opaque et à l'aide d'un éclairage inactinique pour le signal OSL (LEDs à $559 \mathrm{~nm}$ ). Au laboratoire, les échantillons ont été tamisés $(20,40,60 \mu \mathrm{m})$ puis ont subi un traitement à l'acide chlorhydrique $(\mathrm{HCl} 10 \%)$ afin d'éliminer les carbonates, suivi d'eau oxygénée $\left(\mathrm{H}_{2} \mathrm{O}_{2}\right.$ $30 \%$ ) pendant $24 \mathrm{~h}$, afin d'éliminer la matière organique. Pour la fraction granulométrique 40-60 $\mu \mathrm{m}$, les quartz ont été isolés des feldspaths et minéraux lourds grâce à une solution de lithium hétéropolytungstate (LST) préparée à différentes densités. Après ce traitement, l'échantillon LPOSL 1 s'est avéré ne pas contenir suffisamment de quartz ni de feldspaths.

Toutes les mesures de luminescence ont été réalisées sur un lecteur standard Risø TL/OSL-DA-20 (Bøtter-Jensen et al. 2000) équipé d'une source interne bêta ${ }^{90} \mathrm{Sr} / 90 Y$ délivrant un débit de dose de 0,121 $\pm 0,002$ Gy. $\mathrm{s}^{-1}$ à la fraction polyminérale $(20-40 \mu \mathrm{m})$ disposée dans des coupelles, et de 0,159 $\pm 0,003$ Gy. $\mathrm{s}^{-1}$ aux grains de quartz (40-60 $\mu \mathrm{m})$ fixés sur des disques, au moment des mesures.

Pour l'ensemble des échantillons, les contributions externes alpha et bêta ont été calculées à partir des teneurs en radioéléments $\mathrm{K}, \mathrm{U}$ et Th déduites des activités mesurées par spectrométrie gamma haute résolution et bas bruit de fond, sur une portion de sédiment $(\sim 10 \mathrm{~g})$. Les mesures effectuées indiquent des teneurs en radioéléments relativement comparables pour l'ensemble de la séquence stratigraphique (tabl. 4). L'échantillon
LPOSL 3 se distingue par une teneur en uranium de l'ordre de 24 ppm, valeur très inhabituelle et qui pourrait traduire le fait qu'un fragment osseux riche en uranium ait été broyé et mélangé au sédiment fin.

Aux Pradelles, un léger déséquilibre dans la famille de l'uranium a été détecté pour les échantillons LPOSL 4 et 10. L'excès d'activité en éléments de fin de chaîne $\left(\cup_{\text {fin }}\right.$, tabl. 4) par rapport aux éléments de tête de chaîne et à leurs descendants $\left(\cup_{\text {tête, }}\right.$, tabl. 4) peut être dû soit à un appauvrissement en uranium, soit à un enrichissement en radium, en conséquence de la mobilité plus ou moins élevée de ces radioéléments. Les mesures de spectrométrie gamma ne permettent pas de connaître l'origine du déséquilibre observé. Par ailleurs, les analyses sédimentologiques ne mettent pas en évidence de changements environnementaux importants ayant pu bouleverser l'équilibre séculaire. Ainsi, nous faisons l'hypothèse d'un déséquilibre continu dans le temps et postulons que $10 \%$ du débit de dose auquel ont été soumis les échantillons provient des éléments de tête de chaîne et 90 \% de ceux de la fin de chaîne (Aitken et al. 1985), à l'exception de l'échantillon LPOSL 3, pour lequel nous avons considéré que $100 \%$ de la dose émanait de la fin de chaîne.

Des dosimètres en alumine $\mathrm{Al}_{2} \mathrm{O}_{3}: \mathrm{C}$ (voir Mercier et al. 1994 ; Richter et al. 2010) ont été enfouis dans la coupe stratigraphique pendant 243 jours à quelques centimètres de chaque prélèvement de sédiment pour mesurer les doses induites par les rayonnements gamma. Ces doses ont été corrigées de l'effet de l'humidité. La teneur en eau des sédiments à saturation a été mesurée à environ $40 \%$ (en masse). Les observations géologiques ont révélé que la rivière Ligonne formait une anse située sur la rive droite en vis à vis du gouffre et à moins de 16 mètres de la coupe du site, l'eau pouvant s'infiltrer vers le site voire l'inonder lors de phases de crues (Maureille et al. 2008). Du fait de cette 

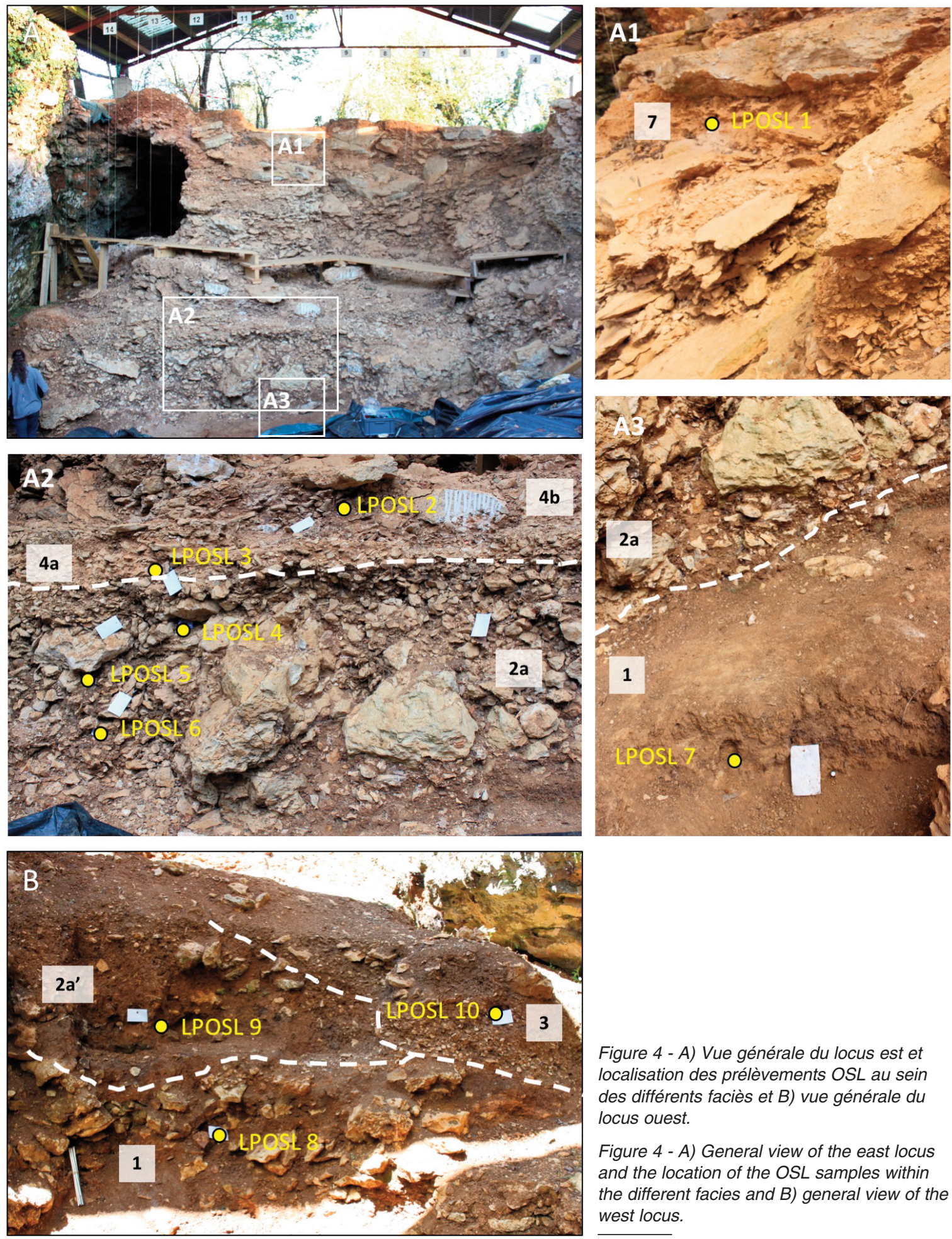

Figure 4 - A) Vue générale du locus est et localisation des prélèvements OSL au sein des différents faciès et $B$ ) vue générale $d u$ locus ouest.

Figure 4 - A) General view of the east locus and the location of the OSL samples within the different facies and $B$ ) general view of the west locus.

configuration, nous avons estimé que le taux d'humidité moyen au cours du temps correspondait à une valeur intermédiaire entre un état totalement sec et un état proche de la saturation, soit $20 \%$. L'incertitude sur cette valeur a été fixée à $50 \%$ de cette valeur $(20 \pm 10 \%$ à $1 \sigma)$ pour tenir compte de la méconnaissance de ce paramètre. Pour les feldspaths, qui contiennent des éléments radioactifs, des teneurs de $10 \pm 2 \%$ en potassium et de $400 \pm 100$ ppm en rubidium ont été prises en compte (Huntley and Hancock 2001) et la contribution de la dose beta a été calculée en tenant compte de la taille des grains considérés. Pour 


\begin{tabular}{|c|c|c|c|c|c|c|c|}
\hline \multirow[b]{2}{*}{ Locus } & \multirow[b]{2}{*}{ Echantillon } & \multirow[b]{2}{*}{ Faciès } & \multicolumn{4}{|c|}{ Radioéléments a } & \multirow[b]{2}{*}{$\begin{array}{c}\text { Rapport } \\
\text { d'activitéb }\end{array}$} \\
\hline & & & K (\%) & $\begin{array}{c}\mathbf{U}_{\text {tête }} \\
\text { (eq. ppm) }\end{array}$ & $\begin{array}{c}\mathbf{U}_{\text {fin }} \\
\text { (eq. ppm) }\end{array}$ & $\begin{array}{c}\text { Th } \\
\text { (ppm) }\end{array}$ & \\
\hline \multirow[t]{6}{*}{ Est } & LPOSL 2 & $4 b$ & $1,36 \pm 0,02$ & $3,93 \pm 0,18$ & $3,94 \pm 0,04$ & $14,04 \pm 0,15$ & 1,00 \\
\hline & LPOSL 3 & $4 a$ & $0,73 \pm 0,02$ & $23,89 \pm 0,33$ & $5,78 \pm 0,05$ & $7,64 \pm 0,12$ & - \\
\hline & LPOSL 4 & $2 \mathrm{a}$ & $2,05 \pm 0,03$ & $3,74 \pm 0,16$ & $4,80 \pm 0,04$ & $16,19 \pm 0,15$ & 1,28 \\
\hline & LPOSL 5 & $2 \mathrm{a}$ & $1,99 \pm 0,03$ & $4,26 \pm 0,16$ & $5,21 \pm 0,04$ & $15,81 \pm 0,14$ & 1,22 \\
\hline & LPOSL 6 & $2 \mathrm{a}$ & $2,01 \pm 0,03$ & $4,75 \pm 0,15$ & $4,73 \pm 0,04$ & $16,33 \pm 0,14$ & 1,00 \\
\hline & LPOSL 7 & 1 & $1,95 \pm 0,03$ & $3,67 \pm 0,15$ & $3,37 \pm 0,03$ & $15,22 \pm 0,13$ & 0,92 \\
\hline \multirow[t]{3}{*}{ Ouest } & LPOSL 10 & 3 & $0,72 \pm 0,01$ & $2,57 \pm 0,14$ & $3,67 \pm 0,04$ & $9,23 \pm 0,10$ & 1,42 \\
\hline & LPOSL 9 & $2 a^{\prime}$ & $1,93 \pm 0,03$ & $3,56 \pm 0,18$ & $3,09 \pm 0,04$ & $16,44 \pm 0,16$ & 0,86 \\
\hline & LPOSL 8 & 1 & $1,61 \pm 0,03$ & $4,50 \pm 0,21$ & $4,59 \pm 0,05$ & $16,13 \pm 0,16$ & 1,02 \\
\hline
\end{tabular}

${ }^{a}$ La teneur $\mathrm{U}_{\text {tête }}$ a été déterminée à partir des émissions de ${ }^{234} \mathrm{Th},{ }^{234} \mathrm{~Pa}$, et ${ }^{235} \mathrm{U}$, et la teneur $\mathrm{U}_{\text {fin }}$ à partir de ${ }^{214} \mathrm{~Pb}$ et ${ }^{214} \mathrm{Bi}$. ${ }^{\mathrm{b}}$ correspond au rapport $\mathrm{U}\left({ }^{226} \mathrm{Ra}\right) / \mathrm{U}\left({ }^{238} \mathrm{U}\right)$.

${ }^{a}$ The $U_{\text {top }}$ content has been determined from the ${ }^{234} \mathrm{Th},{ }^{234} \mathrm{~Pa}$, and ${ }^{235} \mathrm{U}$ emissions, and the $\mathrm{U}_{\text {bottom content from the }}{ }^{214} \mathrm{~Pb}$ and ${ }^{214} \mathrm{Bi}$. ${ }^{\mathrm{b}}$ corresponds to the $\mathrm{U}\left({ }^{226} \mathrm{Ra}\right) / \mathrm{U}\left({ }^{238} \mathrm{U}\right)$ ratio.

Tableau 4 - Synthèse des teneurs en $U$, Th et $K$ des sédiments obtenus à partir de mesures de spectrométrie à bas bruit de fond et haute résolution.

Table 4 - Synthesis of the $U$, Th and $K$ content of the sediments obtained using high resolution low background gamma-ray spectrometry.

chaque fraction minérale étudiée, les débits de dose sont reportés dans le tableau 4.

\section{Datation des feldspaths (fraction polyminérale)}

La présence de feldspaths a été testée sur tous les échantillons par stimulation infrarouge et 6 échantillons ont répondu positivement : LPOSL 2, LPOSL 3, LPOSL 7 pour la partie est du site et LPOSL 8, LPOSL 9, LPOSL 10 pour la partie ouest. La dose équivalente de chacun de ces six échantillons a été déterminée en appliquant le protocole SAR (Single Aliquot Regenerative dose Protocol, voir Murray et Wintle 2003) à une série de 12 aliquotes par échantillon. Le détail du protocole IR50 est reporté dans le tableau 5. Pour la construction de la courbe de croissance en dose, le signal IRSL a été intégré sur les 5 premières secondes et le bruit de fond sur les 10 dernières secondes de stimulation. Les doses équivalentes ont été estimées par interpolation du signal naturel sur la courbe de régénération, ajustée par une fonction exponentielle saturante. L'évaluation du taux de recyclage (en moyenne égal à $0,96 \pm 0,20$ ) et du taux de récupération (toujours $<2 \%$ ) a permis de valider l'application du protocole SAR. La dose équivalente moyenne de chaque échantillon est présentée dans le tableau 6 . Afin de ne pas sous-estimer les âges, les $D_{e}$ mesurées à partir du protocole $\mathrm{IR}_{50}$ ont été corrigées de "l'anomalous fading » (c'est-à-dire, une perte des charges piégées dans le cristal au cours du temps) par la méthode $\mathrm{H}+\mathrm{L}$ (Huntley and Lamothe 2001).

En parallèle, les doses équivalentes pIRIRSL ont été estimées pour trois échantillons (LPOSL 2, LPOSL 3 et LPOSL 7) à partir de 12 aliquotes. Pour certains auteurs (Buylaert et al. 2009 ; Thiel et al. 2011), le signal de pIRIRSL mesuré à $225^{\circ} \mathrm{C}$ (Thomsen et al. 2008) est considéré comme exempt de fading significatif et doit donc fournir une dose équivalente plus élevée que celle obtenue avec le protocole IR50. Ce signal est appelé « pIRIR225 » et le protocole de mesure est présenté dans le tableau 5. Pour les échantillons des Pradelles, la dose équivalente moyenne est reportée dans le tableau 6.

\section{Datation des quartz}

Le signal OSL des quartz des Pradelles est dominé par la composante rapide du signal, qui est celui généralement choisi en matière de datation en raison de ses caractéristiques physiques favorables (bonne stabilité dans le temps, et facilité de remise à zéro lors d'une exposition du grain à la lumière du jour). Nous avons appliqué un protocole standard de SAR pour la détermination de la dose équivalente. Un test de recouvrement de la dose (recovery test) ainsi qu'un test de plateau de dose ont été réalisés suivant différentes conditions de préchauffe pour 18 aliquotes de l'échantillon LPOSL 5. Les résultats (fig. 5) 


\begin{tabular}{|c|c|c|c|}
\hline Etape & $\mathbf{I R}_{\mathbf{5 0}}$ & pIR-IR 225 & OSL \\
\hline 1 & Irradiation & Irradiation & Irradiation \\
\hline 2 & Préchauffe, $60 \mathrm{~s}$ à $250^{\circ} \mathrm{C}$ & Préchauffe, $60 \mathrm{~s}$ à $250^{\circ} \mathrm{C}$ & Préchauffe, $10 \mathrm{~s}$ à $260^{\circ} \mathrm{C}$ \\
\hline 3 & IRSL, $40 \mathrm{~s}$ à $50^{\circ} \mathrm{C}$ & IRSL, 100 s à $50^{\circ} \mathrm{C}$ & OSL, 40 s à $125^{\circ} \mathrm{C}$ \\
\hline 4 & Dose test & IRSL, $100 \mathrm{~s}$ à $225^{\circ} \mathrm{C}$ & Dose test \\
\hline 5 & Préchauffe, $60 \mathrm{~s}$ à $250^{\circ} \mathrm{C}$ & Dose test & Préchauffe, 0 s à $160^{\circ} \mathrm{C}$ \\
\hline 6 & IRSL, 40s à $50^{\circ} \mathrm{C}$ & Préchauffe, $60 \mathrm{~s}$ à $250^{\circ} \mathrm{C}$ & OSL, $40 \mathrm{~s}$ à $125^{\circ} \mathrm{C}$ \\
\hline 7 & IRSL, 40 s à $290^{\circ} \mathrm{C}$ & IRSL, 100 s à $50^{\circ} \mathrm{C}$ & $\mathrm{OSL}, 40$ s à $280^{\circ} \mathrm{C}$ \\
\hline 8 & Retour à l'étape 1 & IRSL, $100 \mathrm{~s}$ à $225^{\circ} \mathrm{C}$ & Retour à l'étape 1 \\
\hline 9 & & IRSL, 40 s à $290^{\circ} \mathrm{C}$ & \\
\hline 10 & & Retour à l'étape 1 & \\
\hline
\end{tabular}

Tableau 5 - Description des protocoles SAR utilisés dans cette étude pour la datation des sédiments des Pradelles.

Table 5 - Description of the SAR protocols used in the analysis of the dating of the Pradelles sediments. indiquent que le protocole SAR mesure avec précision une dose au laboratoire pour des températures comprises entre 220 et $300{ }^{\circ} \mathrm{C}$ (ratio de recovery $=0,9 \pm 0,1 ; n=15$ ). Les échantillons de quartz des Pradelles répondent ainsi à l'ensemble des critères de laboratoire nécessaires pour une estimation fiable de la dose équivalente (taux de recyclage $=1,0 \pm 0,1$; taux de récupération $<1 \%$ ). Pour tous les échantillons de l'ensemble de la séquence, les doses équivalentes ont été déterminées en utilisant une préchauffe fixée à $260^{\circ} \mathrm{C}$, pendant $10 \mathrm{~s}$. La séquence est reportée dans le tableau 5. Les incertitudes sur les doses équivalentes ont été calculées à l'aide du logiciel Analyst (Duller 2007).

Pour une première estimation de la dose équivalente, les échantillons LPOSL 4, 5, 6, 7, 9 et 10 ont été testés à partir de 3 aliquotes. Les doses équivalentes obtenues étant extrêmement faibles pour ces échantillons (tabl. 6), nous avons choisi de focaliser notre étude sur seulement trois

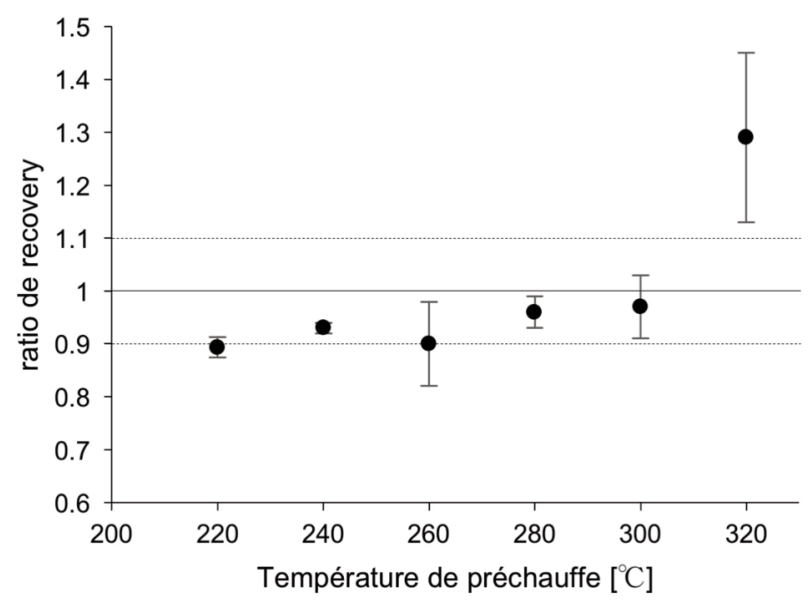

Figure 5 - Test de recovery en fonction de la température de préchauffe pour l'échantillon LPOSL 5. Les aliquotes ont été préalablement remises à zéro dans un simulateur solaire avant irradiation.

Figure 5 - Recovery test as a function of the preheating temperature for the sample LPOSL 5. The aliquots were previously reset in a solar simulator before irradiation. d'entre eux LPOSL 4, 6 et 7. Nous avons alors mesuré 11 à 24 aliquotes pour chacun de ces échantillons.

La dispersion des valeurs de dose équivalente calculée selon le modèle de Galbraith et al. (1999), appelée overdispersion (OD) est particulièrement élevée pour LPSOL $4: 26 \pm 6 \%$ et LPOSL $6: 69 \pm 10 \%$ (tabl. 6 ). Divers facteurs peuvent contribuer à la variabilité des doses mesurées. Deux hypothèses, non exclusives, sont envisagées ici : un environnement microdosimétrique hétérogène des grains de quartz au sein des sédiments ou bien une libération de grains de quartz non blanchis suite à la fantomisation des calcaires. II est important de rappeler ici que chaque dose équivalente mesurée correspond à la moyenne d'une centaine de grains ayant potentiellement eu une histoire dosimétrique différente. Actuellement, aucun modèle connu ne permet de calculer pour des échantillons tels que LPOSL 4 et 6 , une dose équivalente et par conséquent un âge.

Pour l'échantillon LPOSL 7, les courbes de croissance ont été modélisées pour toutes les aliquotes par une fonction de saturation exponentielle (fig. 6) ; de fait, la $D_{e}$ est toujours strictement supérieure à $2 D_{0}$ ( $D_{0}$ étant la dose caractérisant la courbe de la courbe de croissance). L'incertitude sur l'interpolation du signal naturel est alors imprécise et conduit à une distribution asymétrique de l'erreur sur la dose équivalente (Galbraith et Roberts 2012 ; Murray et al. 2002 ; Wintle et Murray 2006). Cette imprécision conduit à une grande incertitude sur l'âge, voire à sa sous-estimation. De fait, nous considérons que l'âge OSL de cet échantillon est un âge minimum.

\section{Discussion des âges obtenus par les méthodes de la luminescence}

Malgré le prélèvement de dix échantillons sur lesquels nous avons entrepris une étude dosimétrique et des mesures de luminescence, seuls trois échantillons se sont révélés

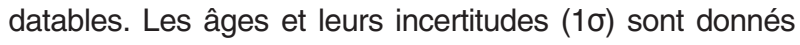
dans le tableau 6.

L'échantillon LPOSL 7 du locus est provenant de I'UL A (faciès 1) a fourni des âges de 74,0 $\pm 3,9$ ka et $53,5 \pm 4,9$ ka 


\begin{tabular}{|c|c|c|c|c|c|c|c|c|c|c|c|c|}
\hline \multirow{2}{*}{ Locus } & \multirow{2}{*}{ Échantillon } & \multirow{2}{*}{ Faciès } & \multirow{2}{*}{$\begin{array}{c}\text { Fraction } \\
(\mu \mathrm{m})\end{array}$} & \multicolumn{4}{|c|}{ Débit de dose $(\mathrm{Gy} / \mathrm{ka})^{\mathrm{a}}$} & \multicolumn{2}{|c|}{$D_{e}(\mathrm{~Gy})$} & \multirow{2}{*}{$\begin{array}{c}\begin{array}{c}\mathrm{g} \\
\text { (\%/décade) }\end{array} \\
\mathrm{IR}_{50}\end{array}$} & \multicolumn{2}{|c|}{ Age (ka) } \\
\hline & & & & alpha & bêta & $\begin{array}{c}\text { gamma } \\
+ \\
\text { cosm. }\end{array}$ & Total & $\mathbf{I R}_{50}$ & pIR-IR 225 & & $\mathbf{I R}_{50}$ & pIR-IR 225 \\
\hline \multirow[t]{3}{*}{ Est } & LPOSL 2 & $4 b$ & $20-40$ & $0,52 \pm 0,11$ & $1,56 \pm 0,01$ & $0,72 \pm 0,09$ & $2,91 \pm 0,13$ & $117 \pm 3$ & $193 \pm 7$ & $2,85 \pm 0,17$ & $52,8 \pm 2,9$ & $66,3 \pm 3,8$ \\
\hline & LPOSL 3 & $4 \mathrm{a}$ & $20-40$ & $0,51 \pm 0,12$ & $1,25 \pm 0,01$ & $0,57 \pm 0,08$ & $2,44 \pm 0,14$ & $93,5 \pm 3$ & $139 \pm 5$ & $3,94 \pm 0,25$ & $57,0 \pm 3,7$ & $57,0 \pm 3,8$ \\
\hline & LPOSL 7 & 1 & $20-40$ & $0,53 \pm 0,11$ & $1,92 \pm 0,03$ & $2,17 \pm 0,41$ & $4,73 \pm 0,41$ & $269 \pm 7$ & $253 \pm 7$ & $3,15 \pm 0,19$ & $74,0 \pm 3,9$ & $53,5 \pm 4,9$ \\
\hline \multirow[t]{3}{*}{ Ouest } & LPOSL 10 & 3 & $40-60$ & $0,34 \pm 0,07$ & $1,03 \pm 0,02$ & $0,60 \pm 0,03$ & $2,15 \pm 0,08$ & $18,1 \pm 2$ & - & $3,29 \pm 0,21$ & $11,2 \pm 0,8$ & - \\
\hline & LPOSL 9 & $2 a^{\prime}$ & $40-60$ & $0,48 \pm 0,11$ & $1,90 \pm 0,03$ & $1,02 \pm 0,07$ & $3,58 \pm 0,12$ & $192 \pm 5$ & - & $3,12 \pm 0,24$ & $71,1 \pm 4,0$ & - \\
\hline & LPOSL 8 & 1 & $40-60$ & $0,51 \pm 0,11$ & $1,80 \pm 0,03$ & $0,42 \pm 0,02$ & $2,91 \pm 0,12$ & $142 \pm 4$ & - & $3,36 \pm 0,28$ & $62,6 \pm 4,6$ & - \\
\hline
\end{tabular}

\begin{tabular}{|c|c|c|c|c|c|c|c|c|c|c|}
\hline \multirow[b]{2}{*}{ Locus } & \multirow[b]{2}{*}{ Échantillon } & \multirow[b]{2}{*}{ Faciès } & \multirow[b]{2}{*}{$\begin{array}{c}\text { Fraction } \\
(\mu \mathrm{m})\end{array}$} & \multicolumn{4}{|c|}{ Débit de dose $(\mathrm{Gy} / \mathrm{ka})^{\mathrm{a}}$} & \multirow[b]{2}{*}{$D_{e}(\mathbf{G y})$} & \multirow[b]{2}{*}{ OD (\%) } & \multirow[b]{2}{*}{$\begin{array}{c}\text { Age OSL } \\
\text { minimum (ka) }\end{array}$} \\
\hline & & & & alpha & bêta & $\begin{array}{c}\text { gamma } \\
+ \\
\text { cosm. }\end{array}$ & Total & & & \\
\hline \multirow[t]{3}{*}{ Est } & LPOSL 4 & $2 \mathrm{a}$ & $20-40$ & $0,30 \pm 0,06$ & $2,13 \pm 0,02$ & $0,65 \pm 0,10$ & $3,07 \pm 0,10$ & $16 \pm 4$ & $26 \pm 6$ & n.d. \\
\hline & LPOSL 6 & $2 \mathrm{a}$ & $20-40$ & $0,30 \pm 0,04$ & $2,10 \pm 0,02$ & $0,73 \pm 0,060$ & $3,13 \pm 0,06$ & $20 \pm 15$ & $69 \pm 10$ & n.d. \\
\hline & LPOSL 7 & 1 & $20-40$ & $0,25 \pm 0,05$ & $1,89 \pm 0,02$ & $2,17 \pm 0,41$ & $4,31 \pm 0,40$ & $307 \pm 36$ & 0 & $71,2 \pm 8.6$ \\
\hline
\end{tabular}

\footnotetext{
${ }^{a}$ Le débit de dose interne de la fraction polyminérale est de 0,112 $\pm 0,020 \mathrm{~Gy} / \mathrm{ka}(20-40 \mu \mathrm{m})$ et 0,182 $\pm 0,020 \mathrm{~Gy} / \mathrm{ka}(40-60 \mu \mathrm{m})$ et celui des quartz a été considéré comme nul.

n.d. : non déterminé

${ }^{\mathrm{a}}$ The internal dose rate for the polymineral fraction is $0.112 \pm 0.020 \mathrm{~Gy} / \mathrm{ka}(20-40 \mu \mathrm{m})$ and $0.182 \pm 0.020 \mathrm{~Gy} / \mathrm{ka}(40-60 \mu \mathrm{m})$; and for the quartz fraction it is considered as negligible.

n.d. : not determined
}

Tableau 6 - Synthèse des données nécessaires aux calculs des âges de la fraction polyminérale et quartz des échantillons des Pradelles.

Table 6 - Synthesis of the data necessary for the calculation of the ages of the polymineral and quartz fraction of Les Pradelles samples.

pour les signaux $I R_{50}$ et pIRIR $R_{225}$, respectivement. L'âge pIRIR 225 apparaît donc plus récent que l'âge $I R_{50}$, en raison probablement d'une sous-estimation du taux de fading pour le signal pIRIR. Pour rappel, aucune mesure de fading n'a été réalisée pour ce signal. L'âge $I_{50}$ est en outre cohérent avec l'âge minimum donné par le signal OSL des quartz $(71,2 \pm 8,6 \mathrm{ka})$, provenant du même échantillon.

Pour l'UL B (faciès 2a), la réponse des quartz n'a pas permis le calcul d'un âge. Du point de vue de la luminescence, aucun des échantillons de quartz issus des niveaux d'occupation humaine n'est exploitable.

L'échantillon de l'UL C (faciès $4 a$ ) a été daté à partir des signaux $\mathrm{IR}_{50}$ et pIRIR 225 de la fraction polyminérale. Les datations obtenues sont respectivement de $57,0 \pm 3,7$ ka et $57,0 \pm 3,8 \mathrm{ka}$. Elles suggèrent une remise à zéro totale de ces signaux lors du dépôt des grains. Pour l'échantillon du faciès $4 b$, les âges $I R_{50}$ et pIRIR ${ }_{225}$ sont respectivement de $52,8 \pm 2,9$ ka et $66,3 \pm 3,8$ ka. Un blanchiment insuffisant du signal pIRIR ${ }_{225}$ peut expliquer la surestimation de son âge par rapport au signal $\mathrm{IR}_{50}$.

Dans le locus ouest, l'échantillon provenant de l'UL A (unité 1) donne un âge de $62,6 \pm 4,6 \mathrm{ka}(1 \sigma)$ cohérent avec les datations obtenues dans la partie est. Pour l'unité 2a', l'échantillon LPOSL 9 donne un âge $\mathrm{IR}_{50}$ de 71,1 \pm 4,0 ka. Dans cette partie du gisement, les données géologiques révèlent que ce niveau correspond au remaniement de dépôts fluviatiles anciens et à des éboulements de l'encaissant calcaire associé à une occupation par des carnivores troglophiles. Nous pensons donc qu'il y a eu mélange de sédiments et d'ossements d'origines et d'âges différents. Cette hypothèse a d'ailleurs déjà été proposée sur la base de l'étude des petits mammifères, qui suggérerait un mélange des vestiges issus du faciès 5 (UL D) et 4b (UL C) (Royer 2013).

Pour le faciès 3 du locus est, une date de 11,2 $\pm 0,8$ ka a été obtenue à partir des feldspaths de l'échantillon LPOSL 10. D'après les études lithostratigraphiques, le faciès 3 du locus est, se situe à l'entrée de la galerie artificielle creusée par les carriers dans les dépôts du 


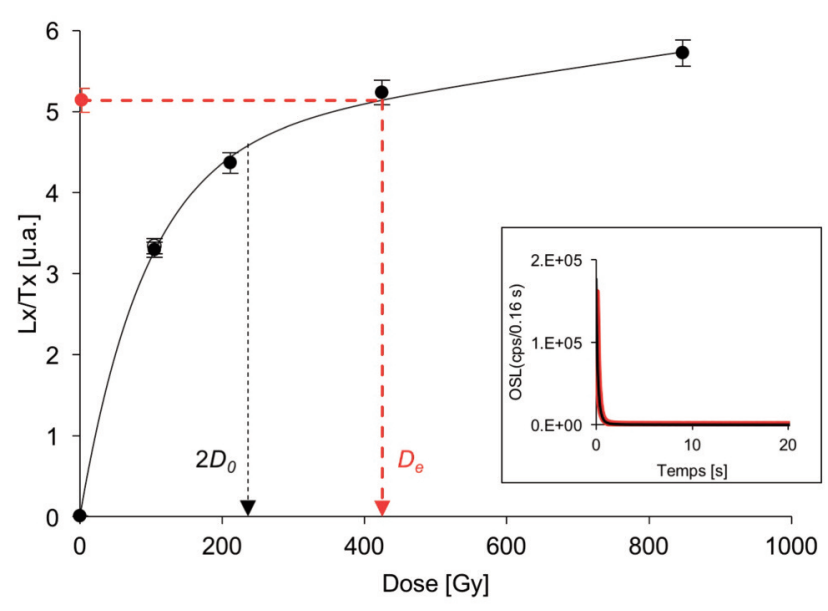

Figure 6 - Courbe de croissance en dose de l'échantillon LPOSL 7. Le signal naturel normalisé est en rouge, et les signaux régénérés sont en noir. La dose équivalente $\left(D_{e}\right)$ se trouve au-delà de la saturation $\left(2 D_{0}\right)$.

Figure 6 - Dose growth curve for the sample LPOSL 7. The normalized natural signal is in red, and the regenerated signals are in black. The equivalent dose $\left(D_{e}\right)$ is beyond the saturation level $\left(2 D_{0}\right)$.

remplissage ancien. Ces dépôts se sont partiellement effondrés ou ont été repris lors de phases pluvieuses par du ruissellement ou des petites coulées de débris. Bien qu'une dent néandertalienne ait été trouvée dans ces sédiments, l'âge apparent obtenu est donc la conséquence du remaniement des sédiments, dont les signaux de luminescence ont pu être remis à zéro, partiellement au moins, lors d'activités récentes.

Au regard des différences notables entre les âges $I R_{50}$ et pIRIR $_{225}$ dues probablement aux effets combinés de l'anomalous fading et d'une remise à zéro insuffisante du signal, nous considérons les âges pIRIR 225 comme seulement informatifs. Ils ne seront donc pas pris en compte dans la synthèse chronologique.

\section{4 - Synthèse chronologique}

Les dates obtenues au travers de ces différentes approches nous permettent de proposer une synthèse chronologique et archéologique de l'évolution de la séquence lithostratigraphique des Pradelles (fig. 7) :

À la base de la séquence, le dépôt d'argiles karstiques stériles (UL $A$, faciès 1 ) se met en place lors de l'écoulement d'une rivière souterraine. Les datations obtenues sur l'échantillon LPOSL 7 indiquent une mise en place des dépôts argileux à la fin du MIS 5 et/ou au début du MIS 4.
La datation U-Th obtenue sur la partie supérieure d'un plancher stalagmitique inclus dans ces dépôts a fourni un âge de 82,0 \pm 0,8 ka, c'est-à-dire du début du MIS 5a (d'après Lisiecki et Raymo 2005), ce qui est cohérent avec une mise en place antérieure au remplissage argileux.

La cavité s'est ensuite ouverte sur l'extérieur et est alors devenue accessible aux animaux et aux humains. Le site est d'abord occupé par des carnivores, comme l'attestent les restes de faune et les coprolithes bien conservés à la base du faciès $2 a$. La présence marquée de campagnols des hauteurs (Microtus gregalis) et les valeurs de $\delta^{15} \mathrm{~N}$ obtenues sur le collagène d'herbivores suggèrent un climat froid et rigoureux (Fizet et al. 1995 ; Royer 2013). Il est donc probable que l'ouverture de la cavité et l'installation des carnivores aient eu lieu pendant le MIS 4, correspondant à de telles conditions climatiques. A quelques dizaines de kilomètres du site des Pradelles, les spéléothèmes de la grotte de Villars (Dordogne) ont enregistré une période extrêmement froide et aride - nommée Villars Cold Phase (D3) - qui s'étale entre environ 68 et 61 ka (Genty et al. 2003, 2010 ; fig. 7) et qui correspond en partie à l'événement climatique d'Heinrich 6 (H6 : 60 - 63 ka ; Sanchez-Goñi et al. 2010, 2013). Malheureusement, l'absence de datation numérique pour le faciès $2 a$ ne permet pas de préciser le lien entre ces événements climatiques, i) l'ouverture de la cavité, et ii) la première installation de carnivores à la base du faciès $2 a$.

Aux Pradelles, les Néandertaliens deviennent ensuite très rapidement les principaux acteurs de l'accumulation des vestiges fauniques pour l'UL $B$ (faciès $2 a$ puis $2 b$ ). Ils semblent occuper le site de façon brève, probablement répétitive et toujours à la même saison - à la fin de la saison chaude ou au début de la saison froide - pour des activités d'exploitation des carcasses de rennes et de traitement des segments les plus riches en ressources alimentaires (Costamagno et al. 2006 ; 2017). Ces groupes sont les artisans d'un Moustérien de type Quina (Bourguignon 1997). Un silex brûlé provenant du faciès $2 b$ a fourni un âge de $58,0 \pm 4,8$ ka correspondant donc à la fin du MIS 4 et au début du MIS 3. Cette date est cohérente avec l'ensemble des datations obtenues pour le technocomplexe Quina dans le Sud-Ouest de la France, qui s'étend du MIS 5 au MIS 3. Ainsi dans le site éponyme de La Quina (Charente), l'industrie est ainsi présente de la fin du MIS 4 jusqu'à la première moitié du MIS 3 (Frouin et al. 2017). Dans le site de Jonzac en Charente (aussi appelé "Chez-Pinaud"), le technocomplexe Quina est daté de la fin du MIS 5 et du début du MIS 4 à 72,7 $\pm 7,9$ ka (Richter et al. 2013). En Dordogne et dans le Lot-et-Garonne (Roc-de-Marsal, Combe-Capelle Bas, Sous-les-Vignes), les datations suggèrent une chronologie plus récente, autour de $45 \mathrm{ka}$ (Valladas et al. 1999, 2003 ; Guérin et al. 2012, 2017). L'ensemble de ces résultats n'est pas sans provoquer d'importantes discussions au sein de notre communauté, ce qui démontre la nécessité de multiplier les datations pour les gisements clés du Paléolithique, ainsi que l'étude systématique des assemblages lithiques. 


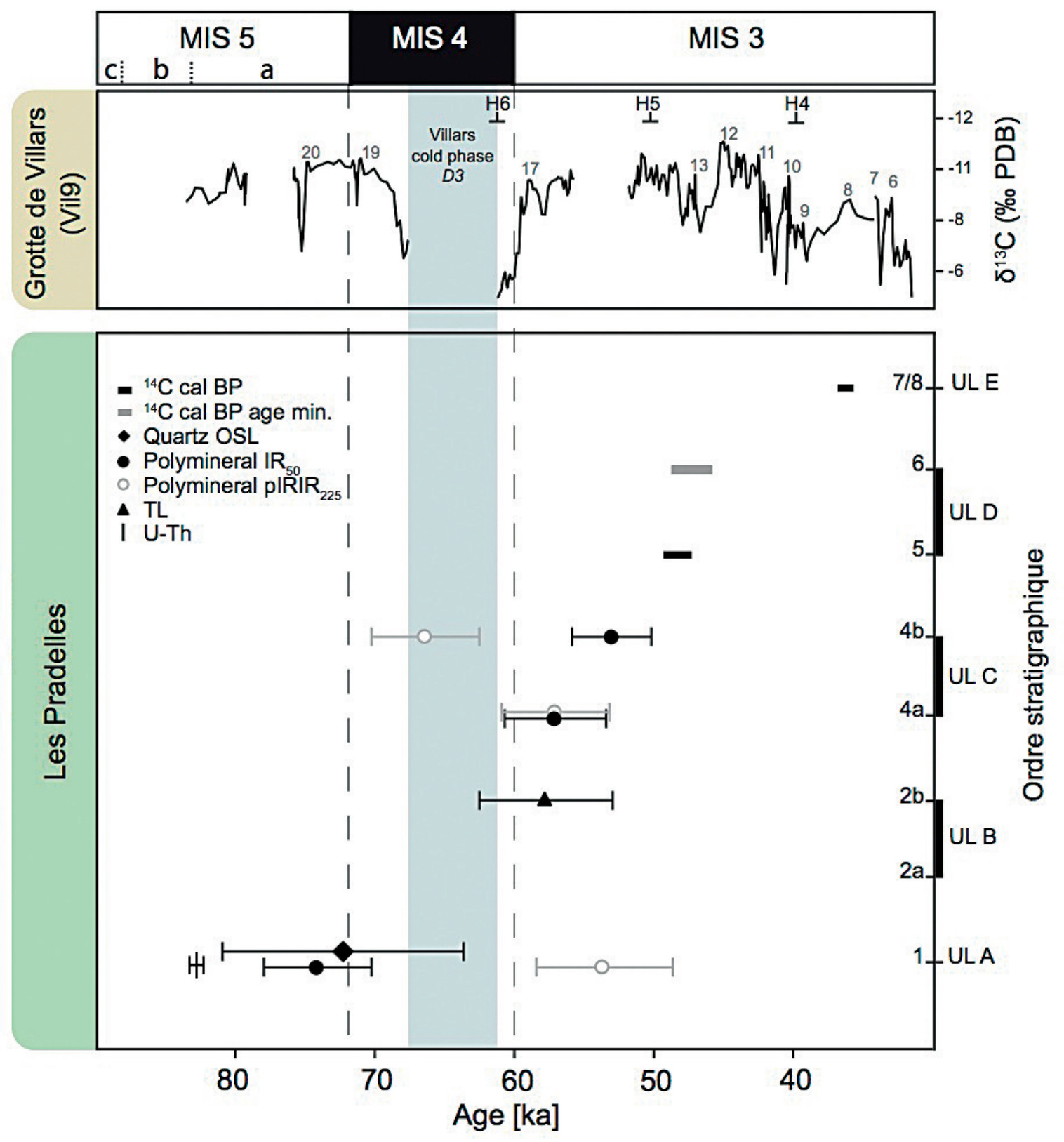

Figure 7 - Synthèse des datations de la partie est du gisement des Pradelles.

Figure 7 - Synthesis of the dates for the east part of Les Pradelles site.

Aux Pradelles, le début de la formation du l'UL C (faciès 4a) est daté d'environ 57 ka et s'inscrit donc dans le même cadre chronologique que la date TL obtenue sur le silex issu de l'UL B (faciès $2 b$ ), soit à la fin du MIS 4 ou au début du MIS 3. II est intéressant de souligner que le spectre faunique du faciès 4a se compose essentiellement de taxons d'environnements froids du Pléistocène supérieur et ne présentent pas de changements significatifs par rapport au faciès précédent $2 b$. $\mathrm{Au}$ sein de la même unité lithostratigraphique, le faciès suivant (4b) donne un âge de 52,8 $\pm 2,9 \mathrm{ka}$. Durant cette période, le gisement se trouvait vraisemblablement dans un environnement ouvert steppique à herbage, probablement parsemé occasionnellement de zones de broussailles et de points d'eau permanents comme l'atteste la présence de grenouilles vertes (Pelophylax kl. esculentus) et de poissons (Maureille et al. 2013). D'un point de vue du spectre des petits mammifères, il est intéressant de constater que des changements importants ont été observés entre le niveau $4 \mathrm{a}$ et $4 \mathrm{~b}$ avec une augmentation de l'abondance des taxons caractéristiques de milieux froids et secs, tels que le lemming à collier (Dicrostonyx torquatus) et la marmotte (Marmota baibacina) (Royer et al. 2013). À ces changements, s'ajoutent également des modifications des compositions isotopiques de l'oxygène mesurées sur les dents de 
rongeurs de ces deux niveaux, qui suggèrent l'hypothèse d'une augmentation importante de l'aridité entre ces deux niveaux (Royer et al. 2013). Sur la base de ces observations, une attribution chronologique à un événement d'Heinrich avait été avancée, privilégiant notamment le HE (Heinrich Event) 6 (Royer et al. 2013). Dans l'état actuel de nos connaissances à l'échelle régionale, les datations obtenues par luminescence ne permettent pas de valider ou d'invalider cette hypothèse. Ces résultats soulignent néanmoins l'importance de continuer nos recherches sur l'impact des changements paléoclimatiques et paléoenvironnementaux sur les zoocénoses au cours du Paléolithique. À la fin de la formation de l'UL C, l'impact des prédateurs sur l'assemblage faunique change : Néandertaliens majoritairement pour le faciès $4 a$, Néandertaliens et carnivores pour le faciès $4 b$.

L'UL D marque une phase intense d'effondrement des parois et du plafond de l'aven (faciès 5 et 6). Ces phénomènes érosifs ne sont pas en mesure de donner des renseignements quant à l'environnement paléoclimatique tout au long du remplissage sédimentaire. En revanche, les dépôts stratifiés de la partie supérieure de l'unité C (faciès 6), dus à des épisodes de solifluxion, témoignent d'un climat périglaciaire (Texier 2009) au cours du MIS 3, possiblement lié au HE 5, hypothèse qui n'est pas contredite par les tentatives de datations radiocarbone. Les carnivores ont été, dès la mise en place du faciès 5 , les principaux prédateurs responsables de l'accumulation des vestiges de faune. Le spectre faunique est caractéristique d'un environnement rigoureux (également confirmé par des résultats préliminaires inédits sur les pollens conservés dans les coprolithes de carnivores) en corrélation avec les données paléoclimatiques (Genty et al. 2010). Dans ces niveaux, la présence humaine est néanmoins attestée par la présence de quelques outils moustériens et des traces de boucherie sur des fragments osseux.

Au-dessus des coulées de solifluxion se trouvent de larges dalles (UL E, faciès 7) traduisant la phase finale d'effondrement du plafond de l'ancienne cavité karstique. Cette partie du site s'arrête alors de fonctionner et est colmatée aux alentours de 38-35 ka cal BP, d'après la datation radiocarbone (fin du MIS 3). Alors que le locus est du gisement est totalement comblé par endroits, le locus ouest reste accessible aux carnivores qui en ont fait leur repaire il y a environ 45-43 ka cal BP. Un autre locus est pour le moment topographiquement distinguable du précédent - la Grotte aux Poules - mais pourrait tout à fait correspondre ou s'inscrire dans la même phase d'occupation du gisement. Malheureusement, il nous est encore difficile d'appréhender la correspondance entre la partie est du gisement et le locus ouest.

\section{5 - Conclusion}

L'ensemble des données géochronologiques disponibles pour le site des Pradelles permet de clarifier la chronologie du gisement, en confirmant d'une part l'attribution de la base de la stratigraphie (UL A) à la fin du MIS 5 et au MIS 4 et, d'autre part, en affinant l'attribution chronologique des occupations humaines et animales successives. Les Néandertaliens, artisans d'un Moustérien de type Quina, ont fréquenté le site des Pradelles entre la fin du MIS 4 et le MIS 3.

Cette étude a été initiée dans le cadre d'un vaste projet visant à définir des jalons chronologiques fiables destinés à préciser l'évolution des cultures néandertaliennes du Paléolithique moyen dans le Sud-Ouest de la France (Guibert et al. 2008 ; Vieillevigne et al. 2008). Comprendre la variabilité des technocomplexes lithiques attribués au Paléolithique moyen revêt en effet une importance capitale pour appréhender la pluralité des organisations socioéconomiques des groupes néandertaliens au sein d'un territoire. Cette problématique a fait l'objet de nombreux questionnements (par exemple Bourguignon et al. 2006 ; Hiscock et al. 2009 ; Delagnes et Rendu 2011 ; Jaubert 2012 ; Faivre et al. 2014, 2017a, 2017b ; Turq et al. 2017). Ce travail sur le gisement des Pradelles permet donc d'avancer dans la compréhension du positionnement chronologique du technocomplexe Quina dans cette partie du Bassin aquitain. II s'ajoute au corpus de sites dont les séquences ont été réétudiées et pour lesquels ce technocomplexe lithique Quina ne semble pas restreint chronologiquement au MIS 4.

\section{Remerciements}

Ce travail a été financé par la Région Aquitaine dans le cadre du projet de recherche "La radioluminescence des feldspaths : un nouvel outil de datation des gisements archéologiques et des séquences quaternaires d'Aquitaine". Les fouilles aux Pradelles et les recherches sur les collections des Pradelles ont fait l'objet de nombreux soutiens dont ceux du Ministère de la Culture, du Service Régional de l'Archéologie (DRAC Poitou-Charentes), du Conseil Général de la Charente, du Conseil Municipal de Marillac-le-Franc (et de ses habitants), de Princeton University, du CNRS, de l'Université de Bordeaux, de l'UMR5199 PACEA, du projet scientifique de la Région Aquitaine : Transitions, d'une société à l'autre processus d'adaptation et de peuplements, convention $n^{\circ} 20051403003 \mathrm{AB}$, du projet scientifique : NéMo (LabEx des Sciences archéologiques de Bordeaux, ANR $n^{\circ}$ ANR-10-LABX-52 / LaScArBx), du projet scientifique de la région Nouvelle Aquitaine : Neanderthalenses Aquitanensis: Territoires, Chronologie, Humanité convention $\mathrm{n}^{\circ}$ 20161R40204-00007349-00007350.

\section{Acknowledgments}

This work was also supported by the Aquitaine Region Council for funding this research, through the program entitled "La radioluminescence des feldspaths: un nouvel outil de datation des gisements archéologiques et des séquences quaternaires d'Aquitaine". Excavations at Les Pradelles and research on archaeological collections have been supported by many fundings : the Ministère de la Culture of France, the Service Régional de l'Archéologie (DRAC Poitou-Charentes), the Conseil Général de la Charente, the Conseil Municipal de Marillac-le-Franc and 
its residents, Princeton University, the CNRS, University of Bordeaux, UMR5199 PACEA, the Research Program of the Aquitaine Region: Transitions, d'une société à l'autre processus d'adaptation et de peuplements, convention $\mathrm{n}^{\circ}$ 20051403003AB and by the Scientific Project: NéMo of the LabEx Sciences archéologiques de Bordeaux, support provided by the ANR - n ${ }^{\circ}$ ANR-10-LABX-52 / LaScArBx, the Research Program of the Nouvelle Aquitaine Region: Neanderthalenses Aquitanensis: Territoires, Chronologie, Humanité convention $\mathrm{n}^{\circ}$ 2016-1R40204-0000734900007350.

\section{Références bibliographiques}

AITKEN M.J. 1985 - Thermoluminescence dating. Academic press (England), $359 \mathrm{p}$.

AITKEN M.J., CLARK P.A. and GAFNEY C.F. 1985 - Beta and gamma gradients. Nuclear Tracks, 10 (4-6), p. 647-653.

BAIZE D., JABIOL B. 1995 - Guide pour la description des sols. Paris : INRA.

BOCHERENS H., FIZET M., MARIOTTI A., LANGEBADE B., VANDERMEERSCH B., BOREL J.-P., BELLON G. 1991 - Isotopic biogeochemistry $\left({ }^{13} \mathrm{C},{ }^{15} \mathrm{~N}\right)$ of fossil vertebrate collagen: implications for the study of fossil food web including Neandertal Man. Journal of Human Evolution, 20, p. 481-492.

BOCHERENS H., DRUCKER D.G., BILLIOU D., PATOUMATHIS M., VANDERMEERSCH B. 2005 - Isotopic evidence for diet and subsistence pattern of the SaintCésaire I Neanderthal: Review and use of a multi-source mixing model. Journal of Human Evolution, 49, p. 71-87.

BOULESTIN B. 2006 - Etait-il cannibale ? Les dossiers de La Recherche, 24, p. 62.

BOURGUIGNON L. 1996 - La conception de débitage Quina. Quaternaria Nova, 6, p. 149166.

BOURGUIGNON L. 1997 - Le Moustérien de type Quina : nouvelle définition d'une entité technique, Thèse de doctorat (Université Paris X-Nanterre).

BOURGUIGNON L. 2001 - Apports de l'expérimentation et de l'analyse techno-morpho-fonctionnelle à la reconnaissance du processus d'aménagement de la retouche Quina. In : Préhistoire et approche expérimentale, sous la direction de L. Bourguignon, I. Ortega et M.C Frère-Sautot, Ed. M. Mergoil (Montagnac), p. 35-66.

BOURGUIGNON L., DELAGNES A., MEIGNEN L. 2006 Systèmes de production lithique, gestion des outillages et territoires au Paléolithique moyen : où se trouve la complexité ? In : Normes techniques et pratiques sociales. De la simplicité des outillages pré et protohistoriques, $\mathrm{XXVl}{ }^{\mathrm{e}}$ rencontres internationales d'archéologie et d'histoire d'Antibes. Dir. L. Astruc, F. Bon, V. Léa, P.-Y. Milcent, S. Philibert. Éditions APDCA, Antibes, 2006. p. 75-86.
BOURGUIGNON L., TURQ A., FAUQUIGNON J. 2013 Lascas de puesta en funcionamiento, de reacondicionamiento y de reciclado de las raederas Quina: Estimación e interpretación tecno-económica. Experimentación en arqueología. Estudio y difusión. Sèrie Monográfica del MACGirona 25-1, p. 229-235.

BØTTER-JENSEN L., BULUR E., DULLER G.A.T., MURRAY A.S. 2000 - Advances in luminescence instrument systems. Radiation Measurements, 32, p. 523-528.

BRONK RAMSEY C. 2009 - Bayesian analysis of radiocarbon dates. Radiocarbon, 51(1), 337-360.

BUYLAERT J.P., MURRAY A.S., THOMSEN K.J., JAIN M. 2009 - Testing the potential of an elevated temperature IRSL signal from K- feldspar. Radiation Measurements, 44, p. 560565 .

COSTAMAGNO S., BEAUVAL C., LANGE-BADRÉ B., VANDERMEERSCH B., MANN A. et MAUREILLE B. 2005 - Homme ou carnivores ? Protocole d'étude d'ensembles osseux mixtes : l'exemple du gisement moustérien des Pradelles (Marillac-le-Franc, Charente). Archaeofauna, 14, p. 43-68.

COSTAMAGNO S., MEIGNEN L., BEAUVAL C., VANDERMEERSCH B. et MAUREILLE B. 2006 - Les Pradelles (Marillac-le-Franc, France): A mousterian reindeer hunting camp? Journal of Anthropological Archaeology, 25, p. 466-484.

COSTAMAGNO S., BOURGUIGNON L., SOULIER M-C., MEIGNEN L., BEAUVAL C., RENDU W., MUSSINI C., MAUREILLE B. 2017 - Bone retouchers and site function in the Quina Mousterian: the case of Les Pradelles (Marillacle-Franc, France). In : J.M. Hudson, A. García Moreno, E. Noack, E. Turner, A. Villaluenga et S. GaudzinskiWindheuser (dir.), "Retouching the Palaeolithic, Becoming Human and the Origins of Bone Tool Technology", Schloss Herrenhausen, Hanover 21 $1^{\text {st }}-23^{\text {rd }}$ October 2015.

DEBÉNATH A. 1974 - Recherches sur les terrains quaternaires charentais et les industries qui leur sont associées, Doctorat d'état, Université Bordeaux I, Faculté des Sciences, 1 t., p. 359-377 et 587-598 p.

DELAGNES A., RENDU W. 2011 - Shifts in Neandertal mobility, technology and subsistence strategies in western France. Journal of Archaeological Science, 38, p. 1771-1738.

DULLER G.A.T. 2007 - Assessing the error on equivalent dose estimates derived from single aliquot regenerative dose measurements. Ancient TL, 25, p. 15-24.

FAIVRE J.-Ph., DISCAMPS E., GRAVINA B., TURQ A., BOURGUIGNON L. 2017a - Cleaning up a Messy Mousterian: How to describe and interpret Late Middle Palaeolithic chrono-cultural variability in Atlantic Europe. Quaternary International, 433, p. 1-3. 
FAIVRE J.-Ph., GRAVINA B., BOURGUIGNON L., DISCAMPS E., TURQ A. 2017b - Late Middle Palaeolithic lithic technocomplexes (MIS 5-3) in the northeastern Aquitaine Basin: Advances and challenges. Quaternary International, 433, p. 116-131.

FAIVRE J.-Ph., DISCAMPS E., GRAVINA B., TURQ A., GUADELLI J.-L., LENOIR M., 2014 - The contribution of lithic production systems to the interpretation of Mousterian industrial variability in south-western France: The example of Combe-Grenal (Dordogne, France). Quaternary International, 350, p. 227-240.

FIZET M., MARIOTTI A., BOCHERENS H., LANGEBADRE B., VANDERMEERSCH B., BOREL J.P., BELLON G. 1995 - Effect of diet, physiology and climate on carbon and nitrogen isotopes of collagen in a late Pleistocene anthropic paleoecosystem (France, Charente, Marillac). Journal of Archaeological Science, 22, p. 67-79.

FROUIN M., LAHAYE C., VALLADAS H., HIGHAM T., DEBENATH A., DELAGNES A., MERCIER N. 2017 Dating the Middle Paleolithic depositis of La Quina Amont (Charente, France) using luminescence methods. Journal of Human evolution, 109: 30-45.

GALBRAITH R.F., ROBERTS R.G., LASLETT G.M., YOSHIDA H., OLLEY J.M. 1999 - Optical dating of single and multiple grains of quartz from Jinmium rock shelter, northern Australia: part I, experimental design and statistical models. Archaeometry, 41, p. 339-364.

GARRALDA M. D. 2008 - Les Néandertaliens : d'autres gestes envers les défunts. In: Vandermeersch B., CleyetMerle J.J, Maureille B., Turq A. Eds., Première Humanité, gestes funéraires des Néandertaliens. Paris. Réunion des Musées Nationaux, p. 42-51.

GARRALDA M. D., GIACOBINI, G., VANDERMEERSCH, B. 2005 - Cut marks on the Combe-Grenal and Marillac Neandertals. A SEM analysis. Anthropologie XLIII, p. 251-271.

GARRALDA M. D., MAUREILLE B., VANDERMEERSCH B. 2014a - Hyperostosis frontalis interna in a Neandertal from Marillac (Charente, France). Journal of Human Evolution, 67, p. 76-84.

GARRALDA M. D., MAUREILLE B., VANDERMEERSCH B. 2014b - Neanderthal Infant and Adult Infracranial Remains from Marillac (Charente, France). American Journal Physical Anthropology, 155, p. 99-113.

GALBRAITH R.F., ROBERTS R.G. 2012 - Statistical aspects of equivalent dose and error calculation and display in OSL dating: An overview and some recommendations. Quaternary Geochronology, 11, p. 1-27.

GENTY D., BLAMART D., OUAHDI R., GILMOUR M., BAKER A., JOUZEL J., VAN-EXTER S. 2003 - Precise dating of Dansgaard-Oeschger climate oscillations in western Europe from stalagmite data. Nature, 421, p. 833 - 837.
GENTY D., COMBOURIEU-NEBOUT N., PEYRON O., BLAMART D., WAINER K., MANSURI F., GHALEB B., ISABELLO L., DORMOY I., VON GRAFENSTEIN U., BONELLI S., LANDAIS A., BRAUER A. 2010 - Isotopic characterization of rapid climatic events during OIS3 and OIS4 in Villars Cave stalagmites (SW-France) and correlation with Atlantic and Mediterranean pollen records. Quaternary Science Reviews, 29(19-20), p. 2799-2820.

GUERIN G., FROUIN M., TUQUOI J., THOMSEN K., GOLDBERG P., ALDEIAS V., LAHAYE C., MERCIER N, GUIBERT P., JAIN M., SANDGATHE D., McPHERRON S.J.P., TURQ A., DIBBLE H.L. 2017 - The complementarity of luminescence dating methods illustrated on the Mousterian sequence of the Roc de Marsal: A series of reindeer-dominated, Quina Mousterian layers dated to MIS 3. Quaternary International, 433, Part B, 102-115.

GUERIN G., DISCAMPS E., LAHAYE C., MERCIER N., GUIBERT P., TURQ A., DIBBLE H. L., McPHERRON J. S. P., SANDGATHE D., GOLDBERG P., JAIN M., THOMSEN K., PATOU-MATHIS M., CASTEL J.-C., SOULIER M. C. 2012 - Multi-method (TL and OSL), multi-material (quartz and flint) dating of the Mousterian site of Roc de Marsal (Dordogne, France): correlating Neanderthal occupations with the climatic variability of MIS 5-3. Journal of Archaeological Science, 39, p. 3071-3084.

GUIBERT P., SCHVOERER M. 1991 - TL dating: low background gamma spectrometry as a tool for the determination of the annual dose. Nuclear Tracks and Radiation Measurements, 18, p. 231-238.

GUIBERT P., VARTANIAN E., BECHTEL F. and SCHVOERER M. 1996 - Non linear approach of TL response to dose: polynomial approximation. Ancient-TL, 14(2), p. 7-14.

GUIBERT P., BECHTEL F., SCHVOERER M., MULLER P., BALESCU S. 1998 - A new method for gamma doserate estimation in heterogeneous media. Radiation Measurements, 9(5), p. 561-572.

GUIBERT P., BECHTEL F., BOURGUIGNON L., BRENET M., COUCHOUD I., DELAGNES A., DELPECH F., DETRAIN L., DUTTINE M., FOLDAGO M., JAUBERT J., LAHAYE C., LENOIR M., MAUREILLE B., TEXIER J.-P., TURQ A., VIEILLEVIGNE E., VILLENEUVE G. 2008 - Une base de données pour la chronologie du Paléolithique moyen dans le Sud-Ouest de la France. In : JAUBERT J., BORDES J.-G., ORTEGA I. (éds), Les sociétés du Paléolithique du grand Sud-Ouest de la France : nouveaux gisements, nouveaux résultats, nouvelles méthodes. Société Préhistorique Française, mém. XLVII, p. 19-40.

HISCOCK P., TURQ A., FAIVRE J.-Ph., BOURGUIGNON L. 2009 - Quina Procurement and Tools Production. In: Lithics Materials and Paleolithic Societies, Edited by B. Adams and B. S. Blades, Wiley-Blackwell, p. 15-24. 
HOFFMANN D.L. 2008 - 230Th isotope measurements of femtogram quantities for U-series dating using multi ion counting (MIC) MC-ICPMS. International Journal of Mass Spectrometry, 275, p. 75-79.

HOFFMANN D.L., PRYTULAK J., RICHARDS D.A., ELLIOTT T., COATH C.D., SMART P.L., SCHOLZ D. 2007 - Procedures for accurate $U$ and Th isotope measurements by high precision MC-ICPMS. International Journal of Mass Spectrometry, 264, p. 97-109.

HUNTLEY D.J., HANCOCK R.G.V. 2001 - The Rb contents of the K-feldspars being measured in optical dating. Ancient TL, 19(2): 43-46.

HUNTLEY D.J., LAMOTHE M. 2001 - Ubiquity of anomalous fading in K-feldspars and the measurement and correction for it in optical dating. Canadian Journal of Earth Sciences, 38, p. 1093-1106.

JAUBERT J. 2012 - Les archéo-séquences du Paléolithique moyen du Sud-Ouest de la France : quel bilan un quart de siècle après François Bordes ?, In : F. Delpech et J. Jaubert (dir.) - François Bordes et la Préhistoire. Actes du colloque international François Bordes, Bordeaux, 22-24 avril 2009, coll. Documents Préhistoriques, Bordeaux, p. 235253.

LISIECKI L.E., RAYMO M.E. 2005 - A PliocenePleistocene stack of 57 globally distributed benthic $\mathrm{d}^{18} \mathrm{O}$ records, Paleoceanography, 20, PA1003.

MAUREILLE B. 2003 - Nouvelles fouilles dans le gisement du Paléolithique moyen des Pradelles (commune de Marillac-leFranc, Charente). Anthropologica et Praehistorica, 114, p. 160161

MAUREILLE B., COSTAMAGNO S., BEAUVAL C., MANN A. E., GARRALDA M. D., MUSSINI C., LAROULANDIE V., RENDU W., ROYER A., SEGUIN G., VANDERMEERSCH B. 2017 - The challenges of identifying partially digested human teeth: first description of Neandertal remains from the Mousterian site of Marillac (Marillac-le-Franc, Charente) and implications for Palaeoanthropological research. Paleo, vol. 28, p. 201-212.

MAUREILLE B., BOCHERENS H., COSTAMAGNO S., GARRALDA M.-D., GEIGL E.-M., GRUN R., LAHAYE Ch., MANN A. E., ROYER A., BENNERR E. A., LACRAMPECUYAUBÈRE Fr., GRANGE T., MUTH X., PENHOUET Y., SOULIER M. C., WILLMES M. 2013 - Les Pradelles. Rapport de fouilles programmées triennales (2010-2012), 281 p.

MAUREILLE B., MANN A. E., BEAUVAL C., BORDES J.-G., BOURGUIGNON L., COSTAMAGNO S., COUCHOUD I., FAUQUIGNON J., GARRALDA M.D., GEIGL E.-M., GRUN R., GUIBERT P., LACRAMPECUYAUBÈRE Fr., LAROULANDIE V., MARQUET J.-CI., MEIGNEN L., MUSSINI C., RENDU W., ROYER A., SEGUIN G., TEXIER J.-P. 2010 - Les Pradelles à Marillacle-Franc (Charente). Fouilles 2001-2007 : nouveaux résultats et synthèse. In : Buisson-Catil, J., Primault, J. (Eds.), Préhistoire entre Vienne et Charente. Hommes et sociétés du Paléolithique. Association des publications chauvinoises, mém. XXXVIII. APC, Chauvigny, p. 145-162.
MAUREILLE B., BOURGUIGNON L., GEIGL E.-M., CHAMPLOT S., GUIBERT P., LAROULANDIE V., MANN A. E., MEIGNEN L., RENDU W., SEGUIN G., VANDERMEERSCH B., GARRALDA M.-D., LEPRETRE B., MUSSINI C., ROYER A. 2008 - Les Pradelles. Rapport de fouilles programmées triennales (2005- 2007), 245 p.

MEIGNEN L. 1988 - Un exemple de comportement technologique différentiel selon les matières premières: Marillac couches 9 et 10, In : Binford L. et Rigaud J.-Ph. (coord.), L'Homme de Néandertal, vol. 4, la technique. Liège : ERAUL, 31, p.7179.

MEIGNEN L., VANDERMEERSCH B. 1986 - Le gisement moustérien de Marillac (Charentes) couche 9 et 10. Caractéristiques des outillages. Économie des matières premières. Préhistoire de Poitou-Charentes. Problèmes Actuels, CTHS Ed., Paris (1986), p. 135-144

MEIGNEN L., COSTAMAGNO S., BEAUVAL C., BOURGUIGNON L., VANDERMEERSCH B. et MAUREILLE B. 2007- Gestion des ressources lithiques au Paléolithique moyen dans une halte de chasse spécialisée sur le renne : Les Pradelles (Marillac-le-Franc, Charente). In : Moncel, M. H., Moigne, A. M., Arzarello, M. \& Peretto, C. (eds.), Aires d'approvisionnement en matières premières et aires d'approvisionnement en ressources alimentaires : approche intégrée des comportements : 127-139. Oxford: Archeopress.

MEIGNEN L., COSTAMAGNO S., BOURGUIGNON L., BEAUVAL C., MAUREILLE B. 2010 - Approche interdisciplinaire d'une halte de chasse au Paléolithique moyen. In : Buisson-Catil J. et Primault J. (éds), Préhistoire entre Vienne et Charente. Hommes et sociétés du Paléolithique. Association des publications chauvinoises, mém. XXXVIII. Chauvigny : APC, p. 163-166.

MERCIER N., VALLADAS H., VALLADAS G. 1994 - A new dosimetric calibration tool. Nuclear Tracks and Radiation Measurements 23, 507-508.

MURRAY A.S., WINTLE A.G. 2003 - The single aliquot regenerative dose protocol: potential for improvements in reliability. Radiation Measurements, 37, p. 3771.

MURRAY A.S., WINTLE A.G., WALLINGA J. 2002 - Dose estimation in using quartz OSL in the non-linear region of the growth curve, Radiation Protection Dosimetry, 101, p. 371-374.

MUSSINI C. 2011 - Les restes humains moustériens des Pradelles (Marillac-le-Franc, Charente, France) : étude morphométrique et réflexions sur un aspect comportemental des Néandertaliens, Thèse de Doctorat, Université de Bordeaux 1, Bordeaux, $478 \mathrm{p}$.

MUSSINI C., BOULESTIN B. et MAUREILLE B. 2010 Cannibalisme versus Anthropophagie. In : BUISSONCATIL J. et PRIMAULT J. dir., Préhistoire entre Vienne et Charente. Hommes et sociétes du Paléolithique, Chauvigny. Association des publications chauvinoises, 38, p.167-168. 
PRESCOTT J.R. et HUTTON J.T. 1994 - Cosmic ray contributions to dose rates for luminescence and ESR dating: large depths and long-term time variations. Radiation Measurements, 23, (2-3), p. 497-500.

REIMER P. 2013 - IntCal13 and Marine13 Radiocarbon Age Calibration Curves $0-50,000$ Years cal BP. Radiocarbon, 55, 4, p. 1869-1887.

RICHARDS D.A., DORALE J.A. 2003 - Uranium-series chronology and environmental applications of speleothems. Reviews in Mineralogy and Geochemistry, 52(1), p. $407-460$.

RICHTER D., DOMBROWSKI H., NEUMAIER S., GUIBERT P., ZINK A. 2010 - Environmental gamma dosimetry for in-situ sediment measurements by OSL of a$\mathrm{Al}_{2} \mathrm{O}_{3}$ :C. Radiation Protection Dosimetry, 141, p. 27-35.

RICHTER D., HUBLIN J.-J., JAUBERT J., McPHERRON S.P., SORESSI M., TEXIER J.-P. 2013 - Thermoluminescence dates for the Middle Palaeolithic site of Chez-Pinaud Jonzac (France). Journal of Archaeological Science, 40, p.1176-1185.

ROYER A. 2013 - Étude paléoenvironnementale et paléoclimatique du Pléistoceène supérieur du Sud-Ouest de la France, à partir d'analyses comparées d'associations fauniques et de biogéochimies effectuées sur les micromammifères : Discussion à partir des sites principalement des Pradelles (Charente), et complémentairement de Bois Roche (Charente) et du Taillis-des- Coteaux (Vienne), Thèse de doctorat.

ROYER A., LECUYER C., MONTUIRE S., ESCARGUEL G., FOUREL F., MANN A. et MAUREILLE B. 2013 - Late Pleistocene (MIS 3-4) climate inferred from micromammal communities and $\delta 180$ of rodents from Les Pradelles, France. Quaternary Research, 80(1), 113-124.

SÁNCHEZ-GOÑI M. F., HARRISON S. P. 2010 - Millennialscale climate variability and vegetation changes during the last Glacial : concepts and terminology. Quaternary Science Reviews, 29 : 2823-2827.

SÁNCHEZ-GOÑI M. F., BARD E., LANDAIS A., ROSSIGNOL L., d'ERRICO F. 2013 - Air-sea temperature decoupling in western Europe during the last interglacialglacial transition. Nature Geoscience, 6 : 837-841.

TEXIER J.-P. 2000 - A propos des processus de formation des sites préhistoriques. Paleo, 12 : 379-386.

TEXIER J.-P. 2009 - Histoire géologique des sites préhistoriques classiques du Périgord, La Micoque, la Grotte Vaufrey, le Pech de l'Aze I et II, La Ferrassie, I'Abri Castanet, Le Flageolet, Laugerie-Haute. Documents préhistoriques $n^{\circ} 25$, Paris, p.193.
TEXIER J.-P., COUCHOUD I. 2007 - L'analyse géologique et stratigraphique. In : Maureille B. (dir.), Les Pradelles (Commune de Marillac-le-Franc, Charente). Rapport triennal, 2007, p. 66-82.

THIEL C., BUYLAERT J.P., MURRAY A, TERHORST B., HOFER I, TSUKAMOTO S., FRECHEN M. 2011 Luminescence dating of the Stratzing loess profile (Austria) Testing the potential of an elevated temperature post-IR IRSL protocol. Quaternary International, 234, p. 23-31.

THOMSEN K.J., MURRAY, A.S., MAYANK J., BØTTERJENSEN L. 2008 - Laboratory fading rates of various luminescence signals from feldspar-rich sediment extracts, Radiation Measurements, 43, p. 1474-1486.

TURQ A., FAIVRE J.-Ph., GRAVINA B., BOURGUIGNON L. 2017 - Management of a Neandertal territory, the example of north-east Aquitain basin: from the facts to the model. Quaternary International, 433, p. 88-101

VALLADAS H., MERCIER N., FALGUERES C., BAHAIN J.-J. 1999 - Contribution des méthodes nucléaires à la chronologie des cultures paléolithiques entre 300000 et 35000 ans BP. Gallia préhistoire, 41, 1, p. 153-166.

VALLADAS H., MERCIER N., JORON J.-L., McPHERRON S., DIBBLE H.L., LENOIR M. 2003 - TL dates for the Middle Paleolithic site of Combe-Capelle Bas, France, Journal of Archaeological Science, 30, p.1443-1450.

VANDERMEERSCH B. 1976 - Les Néandertaliens de Charente. In : LUMLEY de H. (éd.), La préhistoire française, t. I.1. Paris : éditions du CNRS, p. 584-587.

VANDERMEERSCH B. 1980 - Marillac-le-Franc, Gallia Préhistoire, t. 23, fasc. 2, p. 302-303.

VIEILLEVIGNE E., BOURGUIGNON L., ORTEGA I., GUIBERT P. 2008 - Analyse croisée des données chronologiques et des industries lithiques dans le grand sud-ouest de la France (OIS 10 à 3). Paleo, 20:145-165.

WEDEPOHL K.H. 1995 - The composition of the continental-crust. Geochimica et Cosmochimica Acta, 59, p. 1217-1232.

WINTLE A.G., MURRAY A.S. 2006 - A review of quartz optically stimulated luminescence characteristics and their relevance in single-aliquot regeneration dating protocols. Radiation Measurements, 41, p. 369-391. 